\title{
Nrf2 Activation Mediates Antiallodynic Effect of Electroacupuncture on a Rat Model of Complex Regional Pain Syndrome Type-I through Reducing Local Oxidative Stress and Inflammation
}

\author{
Xiaojie Li $\mathbb{D}^{1},{ }^{1}$ Chengyu Yin $\mathbb{D}^{1},{ }^{1}$ Qimiao Hu $\mathbb{D}^{1},{ }^{1}$ Jie Wang $\mathbb{D}^{1},{ }^{1}$ Huimin Nie $\mathbb{D}^{1},{ }^{1}$ Boyu Liu $\mathbb{D}^{1}$, \\ Yan Tai $\odot{ }^{2}$, Junfan Fang $\odot,{ }^{1}$ Junying Du $\left({ }^{1},{ }^{1}\right.$ Xiaomei Shao $\left({ }^{1},{ }^{1}\right.$ Jianqiao Fang $\left({ }^{1},{ }^{1}\right.$ \\ and Boyi Liu $\mathbb{D}^{1}$ \\ ${ }^{1}$ Department of Neurobiology and Acupuncture Research, The Third Clinical Medical College, Zhejiang Chinese Medical University, \\ Key Laboratory of Acupuncture and Neurology of Zhejiang Province, Hangzhou 310053, China \\ ${ }^{2}$ Academy of Chinese Medical Sciences, Zhejiang Chinese Medical University, Hangzhou 310053, China
}

Correspondence should be addressed to Jianqiao Fang; fangjianqiao7532@163.com and Boyi Liu; boyi.liu@foxmail.com

Received 6 October 2021; Accepted 29 December 2021; Published 14 February 2022

Academic Editor: Sachchida Nand Rai

Copyright (C) 2022 Xiaojie Li et al. This is an open access article distributed under the Creative Commons Attribution License, which permits unrestricted use, distribution, and reproduction in any medium, provided the original work is properly cited.

Complex regional pain syndrome type-I (CRPS-I) represents a type of neurovascular condition featured by severe pain in affected extremities. Few treatments have proven effective for CRPS-I. Electroacupuncture (EA) is an effective therapy for pain relief. We explored the mechanism through which EA ameliorates pain in a rat CRPS-I model. The chronic postischemic pain (CPIP) model was established using Sprague-Dawley rats to mimic CRPS-I. We found that oxidative stress-related biological process was among the predominant biological processes in affected hindpaw of CPIP rats. Oxidative stress occurred primarily in local hindpaw but not in the spinal cord or serum of model rats. Antioxidant N-acetyl cysteine (NAC) attenuated mechanical allodynia and spinal glia overactivation in CPIP model rats, whereas locally increasing oxidative stress is sufficient to induce chronic pain and spinal glia overactivation in naive rats. EA exerted remarkable antiallodynia on CPIP rats by reducing local oxidative stress via enhancing nuclear factor erythroid 2-related factor 2 (Nrf2) expression. Pharmacological blocking Nrf2 abolished antioxidative and antiallodynic effects of EA. EA reduced spinal glia overactivation, attenuated the upregulation of inflammatory cytokines, reduced the enhanced TRPA1 channel activity in dorsal root ganglion neurons innervating the hindpaws, and improved blood flow dysfunction in hindpaws of CPIP rats, all of which were mimicked by NAC treatment. Thus, we identified local oxidative injury as an important contributor to pathogenesis of animal CRPS-I model. EA targets local oxidative injury by enhancing endogenous Nrf2-mediated antioxidative mechanism to relieve pain and inflammation. Our study indicates EA can be an alternative option for CRPS-I management.

\section{Introduction}

Complex regional pain syndrome type-I (CRPS-I) is a debilitating pain condition that usually affects the extremities of the patients [1]. It can be triggered by an initial injury, including surgery, ischemia, and fracture, and can progress into a chronic stage that significantly affects the patients' daily activity $[2,3]$. Clinical manifestations of CRPS-I include spontane- ous pain, thermal/mechanical pain hypersensitivities, limb edema, microvascular injury, and blood flow abnormality [4]. Pain is among the clinical manifestations that mostly affect the patients, both physically and mentally $[5,6]$.

To study CRPS-I mechanisms, a rat chronic postischemic pain (CPIP) model was developed by applying prolonged ischemia, followed with reperfusion to the hind limb [7]. The CPIP model exhibited a number of key 
features that mimic clinical symptoms of CRPS-I, such as chronic thermal, mechanical, and chemical pain hypersensitivities in affected hind limbs, followed with microvascular injury and abnormalities in regional blood flow $[8,9]$. The rat CPIP model has been widely applied for mechanistic studies of CRPS-I. Recently, we and others contributed to this field by identifying a number of pivotal mechanisms contributing to CRPS-I pathogenesis, including peripheral NMDA and TRPA1/TRPV1 channels, the chemokine CXCL12/CXCR4 signaling, NLRP3 inflammasome, and CSF1 in the spinal cord [10-14].

Peripheral tissues are densely innervated by free nerve endings that are projected from peripheral sensory ganglion (e.g., dorsal root ganglion). These free nerve endings act as nociceptors to detect neuroactive substances released from inflammatory cells or injured tissues, which trigger pain and/or produce pain sensitization. CRPS-I is related with microcirculation impairment associated with local tissue inflammation in the affected limb [15]. Therefore, pathophysiological changes in local tissue may play important roles in mediating the pathogenesis of CRPS-I.

Clinical trials failed to prove the beneficial effects of a number of conventional interventions, which makes CRPSI a difficult-to-treat condition in the clinic [16, 17]. Therefore, there is an urgent need for alternative therapeutic options for CRPS-I. One promising alternative therapeutic option for CRPS-I is electroacupuncture (EA). EA combines traditional manual acupuncture with modern electrotherapy and has been widely used for relieving many kinds of pain conditions. Although the efficacy and safety of EA in relieving CRPS-I in clinic have been summarized recently [18], the detailed mechanisms underlying EA's therapeutic effects on CRPS-I still remain largely unexplored.

In this study, in order to explore the mechanisms underlying CRPS-I, we performed an unbiased RNA-Seq in local hindpaw tissues to explore potential substances or biological processes that are affected during CRPS-I. We then explored the potential involvement of these substances or processes in mediating CRPS-I and further examined whether EA exerts therapeutic effects on CRPS-I through acting on these mechanisms by means of biochemistry, pharmacological and behavioral testing, and neuronal functional imaging.

\section{Materials and Methods}

2.1. Animals. Sprague-Dawley rats (male and female, 3-4 months of age) were bought from Shanghai Laboratory Animal Center of China. All animals were housed in Zhejiang Chinese Medical University Laboratory Animal Center (5 animals/cage, $12 \mathrm{~h}$ dark-light cycle, $24 \pm 2^{\circ} \mathrm{C}$ ). Animals were given free access to water and food. All animals were allowed at least 1 week to adjust to the new breeding facility before any test. Animals were randomly allocated using random number tables. A total of 180 rats were used in this study. The group size in our experiments was chosen based upon our previous experience and studies using similar experimental protocols. All experimental procedures were carried out in accordance with the National Institutes of Health Guide for the Care and Use of Laboratory Animals (NIH
Publications No. 8023, revised 1978), and the procedures were all approved by Animal Ethics Committee, Zhejiang Chinese Medical University (\# ZSLL2017-183).

2.2. Animal Model of CRPS-I Establishment. Chronic postischemia pain model was set up via imposing ischemia and reperfusion to the hind limb of the rat $[7,8]$. The rats were anesthetized by injecting $50 \mathrm{mg} / \mathrm{kg}$ (i.p.) sodium phenobarbital.

$7 / 32(5 \mathrm{~mm})$ internal diameter $\mathrm{O}$-ring was used to ligate the hind limb at a position near ankle joint for $3 \mathrm{~h}$. Three hours later, the O-ring was removed. The control group of rats was treated with the same anesthesia steps but without O-ring ligature. No analgesic was provided during model establishment since it could feasibly interfere with the development of the pain state of this animal model.

2.3. Paw Edema Measurement. The edema of the hindpaw was measured by the digital caliper as previously reported [19]. The percent increase in paw thickness was used to indicate the paw edema. Three measurements were taken for each rat, and the average value was obtained thereafter.

2.4. Mechanical Allodynia. Mechanical allodynia was measured by methods described before [20]. Briefly, each rat was allowed to acclimate to testing conditions for $30 \mathrm{~min}$ beforehand. Mechanical allodynia was measured by applying von Frey filaments to the midplantar surface of the rat's hindpaw. The "Up and Down" method was used for measurement and calculation of $50 \%$ paw withdrawal threshold $[21,22]$. The experimenter for behavioral test was blinded during the allocation, the conduct of the experiment, the outcome assessment, and the data analysis procedures.

2.5. Drug Treatment. N-Acetyl cysteine (NAC, Beyotime Biotechnology, China) was diluted in PBS and daily administered through intraperitoneal injection $(200 \mathrm{mg} / \mathrm{kg}$, i.p.) $30 \mathrm{~min}$ ahead of behavior test. The dosage of NAC is based upon previous literatures $[23,24] . \mathrm{H}_{2} \mathrm{O}_{2}$ was diluted in PBS and injected $(100 \mathrm{nmol} / 50 \mu \mathrm{l})$ to dorsal region of hindpaw. The control group of rats was injected with vehicle (PBS) only. ML385 (Tocris, USA) was dissolved in DMSO and further diluted in PBS for local hindpaw injection $(400 \mu \mathrm{g} /$ site in $50 \mu \mathrm{l}$ volume). ML385 was injected $30 \mathrm{~min}$ before each EA intervention.

2.6. EA Procedure. The same EA procedure was adopted in the present study from our recent reports, with some minor modifications [10]. Briefly, the animals were immobilized gently with a self-made retainer. Four acupuncture needles were inserted into bilateral ST36 and BL60 acupoints (with a depth of around $5 \mathrm{~mm}$ ). The HANS-200A Acupoint Nerve Stimulator (Huawei Co., Ltd., China) was used to connect the acupuncture needles. $2 / 100 \mathrm{~Hz}$ alternative square wave with intensities from $0.5-1.5 \mathrm{~mA}$ was administered for 30 min per session. For animals receiving sham EA intervention, the acupuncture needle was only subcutaneously inserted (at a depth of $1 \mathrm{~mm}$ ) into bilateral BL60 and ST36, and no electrical stimulation was applied. The EA or sham EA interventions were performed on animals on a daily basis for 7 days. 
2.7. Tissue Collection and RNA Extraction. CPIP or control group rats were anesthetized deeply using sodium pentobarbital at dosage of $40 \mathrm{mg} / \mathrm{kg}$. The animals were then perfused with $0.9 \%$ saline with $4^{\circ} \mathrm{C}$. Then, the ipsilateral hindpaw skin was harvested and immediately preserved in RNAlater solution (Thermo Fisher, USA). TRIzol reagent (Thermo Fisher, USA) with DNase I was used for total RNA extraction. The concentrations and the purities of skin samples were evaluated using the NanoDrop Spectrophotometer (NanoDrop Products, USA). RNA integrity number (RIN) was determined by the Agilent TapeStation System (Agilent Technologies, USA).

2.8. RNA-Seq Library Establishment and RNA-Seq. Total mRNAs from 3 rats of the CPIP and control groups were isolated and used to construct sequencing libraries. The mRNA molecules were purified from total RNA using oligo (dT)-attached magnetic beads. The mRNAs were fragmented into small pieces using fragmentation reagent. Firststrand cDNA was generated using random hexamerprimed reverse transcription and then was followed by a second-strand cDNA synthesis. The synthesized cDNA was subjected to end-repair and then was $3^{\prime}$ adenylated. Adaptors were ligated to the ends of these $3^{\prime}$ adenylated cDNA fragments. This process is to amplify the cDNA fragments with adaptors from the previous step. PCR products are purified with the SPRI beads and dissolved in EB solution. The double-stranded PCR products were heat denatured and circularized by the splint oligosequence. The singlestrand circle DNA (ssCir DNA) was formatted as the final library. Library was validating on the Agilent Technologies 2100 bioanalyzer. The library was amplified with phi29 to make DNA nanoball (DNB) which has more than 300 copies of one molecular. The DNBs were loaded into the patterned nanoarray, and single-end 50 base reads were generated in the way of sequencing by synthesis. Finally, the fragments were enriched by PCR amplification to construct a library ready for sequencing using BGISEQ-500 by BGI (Shenzhen, China).

2.9. Bioinformatics Analysis. Primary sequencing data produced by RNA-Seq (raw reads) were subjected to quality control (QC). Raw data were filtered into clean reads by internal software SOAPnuke (version 1.5.2) as follows: remove reads in which unknown bases $(\mathrm{N})$ are more than $10 \%$; remove reads with adaptors; remove low-quality reads (we define the low-quality read as the percentage of base in which quality is lesser than 15 and greater than $50 \%$ in a read). QC of alignment was performed to determine if resequencing was needed. If the alignment result passed QC, downstream analysis includes gene expression, differentially expressed genes, and cluster analysis.

2.10. Cluster Analysis and Screening of Differentially Expressed Genes. Distances of expressed genes were calculated using the Euclidean method [25]. The sum of the squared deviation algorithm was used to calculate distance. The cluster analysis and heat map visualization of gene expression patterns were performed using the "pheatmap" package in the $\mathrm{R}$ software of Bioconductor. Differentially expressed mRNAs with statistical significance were identified through Scatter Plot filtering as we reported before [26]. The threshold required for the results to be considered significant was as follows: $q$ value $\leq 0.05$ and absolute value of $\mid \log _{2}$ (fold change) $\mid \geq 0.585$.

2.11. Determination of Oxidant/Antioxidant Status. Ipsilateral hindpaw tissues from each group were collected. The samples were then homogenized, followed by centrifugation at $3,000 \mathrm{rpm}$ for $15 \mathrm{~min}$. Supernatant was collected for superoxide dismutase (SOD), reduced glutathione (GSH), malondialdehyde (MDA), and hydrogen peroxide $\left(\mathrm{H}_{2} \mathrm{O}_{2}\right)$ assay as we described before [23, 27].

2.12. $q P C R$ Testing and Analysis. RNA from the ipsilateral hindpaw skin samples was extracted by TRIzol reagent (Thermo Fisher, USA). 1000 ng of total RNA from hindpaw tissues was reversely transcribed with Takara PrimeScript ${ }^{\mathrm{TM}}$ Master Mix kit to generate cDNAs. qPCR reactions were performed using the FastStart Universal SYBR ${ }^{\circledR}$ Green Master PCR kit (Takara Bio Inc., China). Three replicates were performed for each sample. The gene expression level was normalized to $\beta$-actin, the housekeeping control gene. CFX96 Real-Time System software was used for CT value determination and analysis. Relative mRNA expression levels were determined by the well-established $2^{\Delta \Delta} \mathrm{CT}$ methodology. For detailed information regarding the sequences for the primers, please check our Suppl. Table 1.

2.13. Immunofluorescence Staining. Rats were anesthetized and perfused transcardially with $4^{\circ} \mathrm{C}$ saline followed by $4 \%$ fresh paraformaldehyde. The ipsilateral hindpaw skin and the ipsilateral spinal cord were harvested, fixed for $4 \mathrm{~h}$ in $4 \%$ paraformaldehyde, and gradient dehydrated in sucrose solution $(30 \%)$ overnight at $4^{\circ} \mathrm{C}$. Samples embedded in a frozen microtome (Thermo NX50, Thermo Fisher, USA) were cut into frozen sections with the thickness for $14 \mu \mathrm{m}$ of hindpaw skin and $25 \mu \mathrm{m}$ of the spinal cord. Then, samples were mounted onto gelatin-coated glass slides for immunofluorescence. The sample sections were blocked in normal donkey serum (5\%) in Tris-buffered saline with Tween (TBST) for $1 \mathrm{~h}$ at room temperature and then incubated with corresponding primary antibodies overnight at $4^{\circ} \mathrm{C}$. The primary antibody was applied to immunostain the hindpaw skin sample sections: mouse anti-8-OHG (1:800, ab62623, Abcam), and the primary antibodies were applied to immunostain spinal cord sample sections: rabbit anti-c-Fos ( $1: 800$, \#2250, CST), mouse anti-GFAP ( $1: 1000$, \#c9205, Sigma), and mouse anti-OX42 (1:1000, \#ab1211, Abcam). These antibodies have successfully been used in previous literatures for immunofluorescence staining of corresponding targets [28-31]. Then, the sample sections were labeled with the fluorescent secondary antibodies (Cy3-, Cy5-, or FITCconjugated) for $1 \mathrm{~h}$ at room temperature after washing in the dark. The pictures of sample sections were captured by a laser scanning confocal microscope (Nikon A1R, Japan). Images were captured with uniformed settings, and experimenters were blinded to treatment groups for image 
quantification. Three sections from each animal were selected, averaged, and then compared among groups as described previously in our studies $[32,33]$.

2.14. Western Blotting. The procedures are described in our previous study [27]. Briefly, the ipsilateral hindpaw tissues were collected quickly on ice after the rats being anesthetized with sodium pentobarbital $(40 \mathrm{mg} / \mathrm{kg}$, i.p.). The tissues were then homogenized in RIPA lysis buffer and centrifuged at $15,000 \mathrm{rpm}$ for $15 \mathrm{~min}$ at $4^{\circ} \mathrm{C}$. The supernatants were collected and measured the protein concentration using a BCA protein assay kit (Thermo Fisher, USA). Equal amounts of protein samples $(10 \mu \mathrm{g})$ were electrophoresed on $5 \%-12 \%$ SDSpolyacrylamide gels and transferred onto PVDF membranes (Bio-Rad, USA). After the membranes were blocked by $5 \%$ nonfat milk in TBST solution for $1 \mathrm{~h}$ at room temperature, the primary antibodies were applied overnight at $4{ }^{\circ} \mathrm{C}$ and the HRP-coupled secondary antibodies were applied for $2 \mathrm{~h}$ at room temperature. The antibodies used in the present study were listed as follows: $\beta$-actin $(1: 5000$, mouse monoclonal, \#ab20272, Abcam), 4-hydroxynonenal (4-HNE, $1: 800$, rabbit polyclonal, \#ab46545, Abcam), and nuclear factor erythroid 2related factor 2 (Nrf2, 1:1000, \#rabbit polyclonal, R-1312, HUABIO). 4-HNE and Nrf2 antibodies have been successfully used in previous studies for protein detection [23, 34]. The expression levels of targeted protein are normalized to the density of $\beta$-actin. The original full blot images can be found in Suppl. Fig. 1.

2.15. DRG Harvest and $\mathrm{Ca}^{2+}$ Imaging. $\mathrm{Ca}^{2+}$ imaging was performed as we described before [35]. Briefly, DRG were isolated from ipsilateral lumbar L4-L6 segment from each rat. Samples were not pooled from multiple rats, and a total of 15 rats were used in this study. The DRG were dissociated by collagenase $(1 \mathrm{mg} / \mathrm{ml})$ and dispase $(2 \mathrm{mg} / \mathrm{ml})$ (Gibco, Thermo Fisher) and incubated in DMEM $+10 \%$ FBS and used 4-6h after being harvested. Cells were first washed 3 times with the loading buffer which contains $140 \mathrm{mM} \mathrm{NaCl}$, $5 \mathrm{mM} \mathrm{KCl}, 2 \mathrm{mM} \mathrm{CaCl}, 2 \mathrm{mM} \mathrm{MgCl}$, and $10 \mathrm{mM}$ HEPES ( $\mathrm{pH} 7.4$ adjusted with $\mathrm{NaOH}$ ). Then, the cells were incubated with $10 \mu \mathrm{M}$ Fura $2-\mathrm{AM}$ at $37^{\circ} \mathrm{C}$ for $30 \mathrm{~min}$ in the loading buffer. After the incubation, the cells were rinsed with loading buffer for 3 times and rest for another $30 \mathrm{~min}$ at $37^{\circ} \mathrm{C}$ in the dark. Then, the cells were subject to $\mathrm{Ca}^{2+}$ imaging procedure. $\mathrm{Ca}^{2+}$ imaging was undertaken by a Nikon ECLIPSE Ti-S microscope. Orca Flash 4.0 CCD camera (Hamamatsu, Japan) was used for capturing images. Polychrome V monochromator (Till Photonics, USA) was used as the light source to generate 340 and $380 \mathrm{~nm}$ ultraviolet light. The fluorescence images were further analyzed and processed by the ImageJ software. A criterion was set up to distinguish whether a cell is responsive in $\mathrm{Ca}^{2+}$ imaging test, namely, when the peak $\mathrm{Ca}^{2+}$ signal of the cell reaches over $20 \%$ of the baseline value, then the cell is considered as a positively responding cell $[36,37]$.

2.16. Regional Blood Flow Measurement by Laser Doppler. The rats were anesthetized with isoflurane before laser Doppler measurement. Anesthesia is first achieved with 3-
$4 \%$ isoflurane supplied with oxygen, and then, the anesthesia was further maintained with constant 1-2\% isoflurane supplied with oxygen. The rats were laid on a thermal pad maintained constantly at $37^{\circ} \mathrm{C}$. The PeriFlux System 5000 Laser Doppler Flow Meter (Perimed, Sweden) was used to measure regional blood flow in the affected hindpaws of rats. The whole area of the hindpaw was defined as the region of interest, and the blood flow was then calculated using the software provided by PeriFlux System (Perimed, Sweden).

2.17. Statistical Analysis. SPSS 19.0 (IBM Corp., USA) was used for all statistical analysis through the study. All data were included in data analysis and presentation. Data was presented as the mean \pm SEM. The complete statistical results (including mean, SEM, SD, and confidence interval) are presented in Suppl. Table 2. To compare data between 2 groups, Student's t-test was applied for statistical analysis. To compare data among 3 or more groups, oneway or two-way analysis of variance (ANOVA) with Tukey's post hoc test was applied for statistical analysis. In cases when the data was nonnormally distributed (tested by Kolmogorov-Smirnov test), a nonparametric test (e.g., Mann-Whitney test) was used for analysis. Statistical significance was accepted at a level of $p<0.05$.

\section{Results}

3.1. RNA-Seq Revealed Oxidation-Reduction Process Is Markedly Affected in Hindpaw Tissues of a Rat Model of CRPS-I. The rat CPIP model that mimics human CRPS-I was established according to the methods before $[7,8]$. The ligated hindpaws of the model rats displayed obvious signs of tissue cyanosis and edema as shown in Figures 1(a) and 1(b) during ligation. The edema lasted over two days and returned back to normal (Figure 1(c)). Remarkable bilateral mechanical pain allodynia was observed in model rats compared with control rats that lasted until the endpoint of our study ( $>10$ days) (Figures 1(d)-1(g)). We aimed to explore potential genes or biological process involved in mediating the pain response of CPIP model rats. Therefore, we performed gene expression profiling by RNA-Seq to explore potential gene expression changes in ipsilateral hindpaw tissues from CPIP rats vs. control rats. The ipsilateral hindpaw tissues were collected $7 \mathrm{~d}$ after CPIP model establishment. We successfully extracted high-quality RNA from hindpaw tissues for RNA-Seq analysis (Suppl. Fig 2). A total of 226 (upregulated) and 192 genes (downregulated), amounting to $1.48 \%$ and $1.26 \%$ of all genes being sequenced $(15,251)$, respectively, were identified to be differentially expressed genes (DEGs) in hindpaw tissues from CPIP rats vs. control rats. We overlaid these DEGs in a heat map and performed cluster analysis to further examine data consistency between and within groups (Figure 2(a)). The cluster analysis indicated that the dataset within the CPIP or control groups exhibited high consistency, whereas the two groups are clearly separated (Figure 2(a)). We next performed Gene Ontology (GO) analysis of the DEGs we have identified. Figure 2(b) 


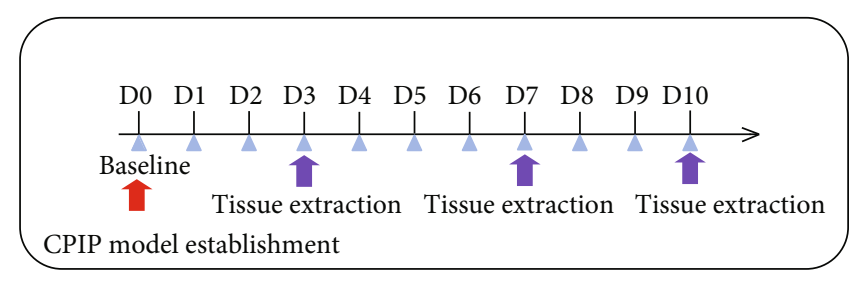

$\triangle$ Behavior test

(a)

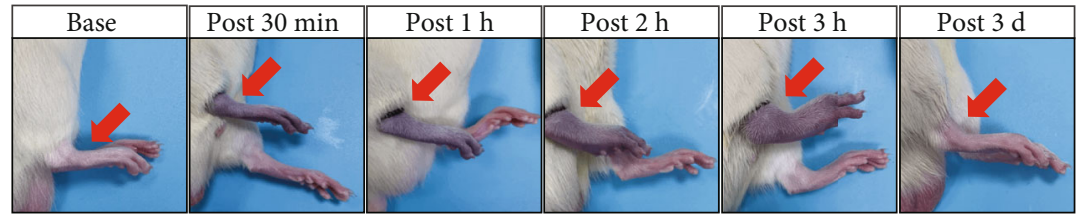

(b)

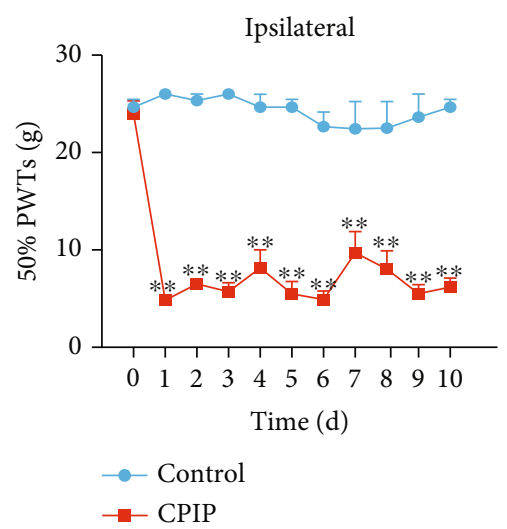

(d)

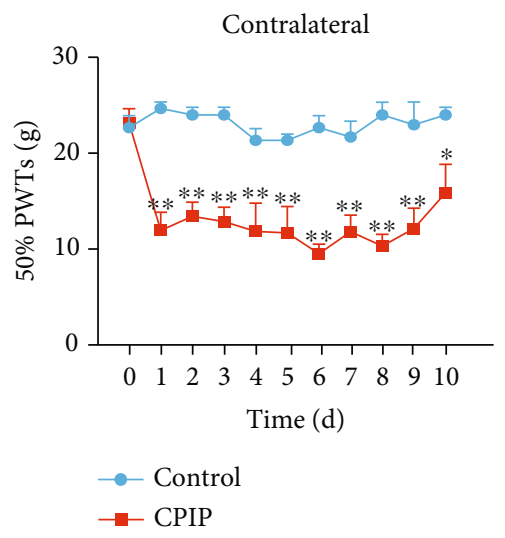

(f)

(e)

(g)

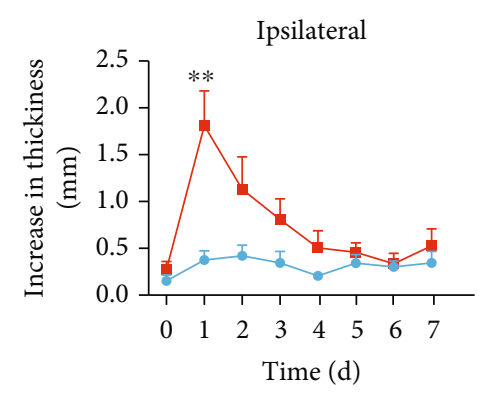

- Control

$\rightarrow-$ CPIP

(c)
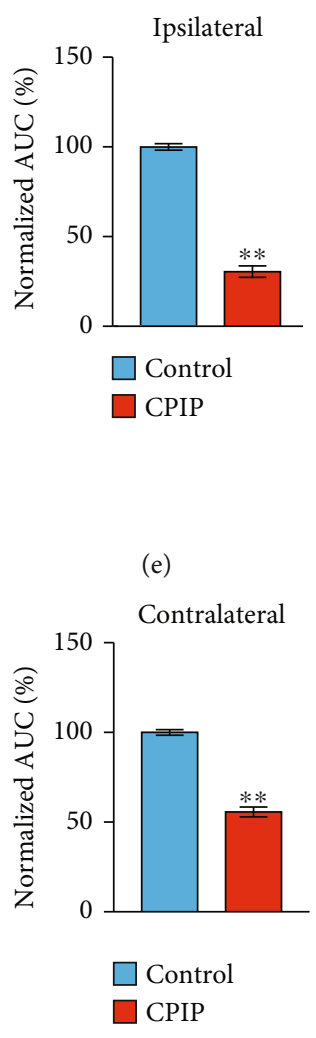

FIgURE 1: The rat CPIP model showed persistent bilateral mechanical allodynia in the hindpaw. (a) Experimental protocol for establishing the rat chronic postischemic pain (CPIP) model. (b) Representative pictures showing the rat's hindpaw during and after CPIP model establishment. The red arrow indicates the paw with the O-ring treatment (ipsilateral side). (c) Paw thickness evaluation of control and CPIP model rats of ipsilateral side. (d) 50\% paw withdrawal threshold (PWT) measurement of ipsilateral hindpaw. (e) Summary of the normalized area under the curve (AUC) as in (d). (f) 50\% PWT measurement of contralateral hindpaw. (g) Summary of the normalized area under the curve (AUC) as in (f). $n=6$ rats/group. ${ }^{* *} p<0.01$ and ${ }^{*} p<0.05$ vs. the control group. 


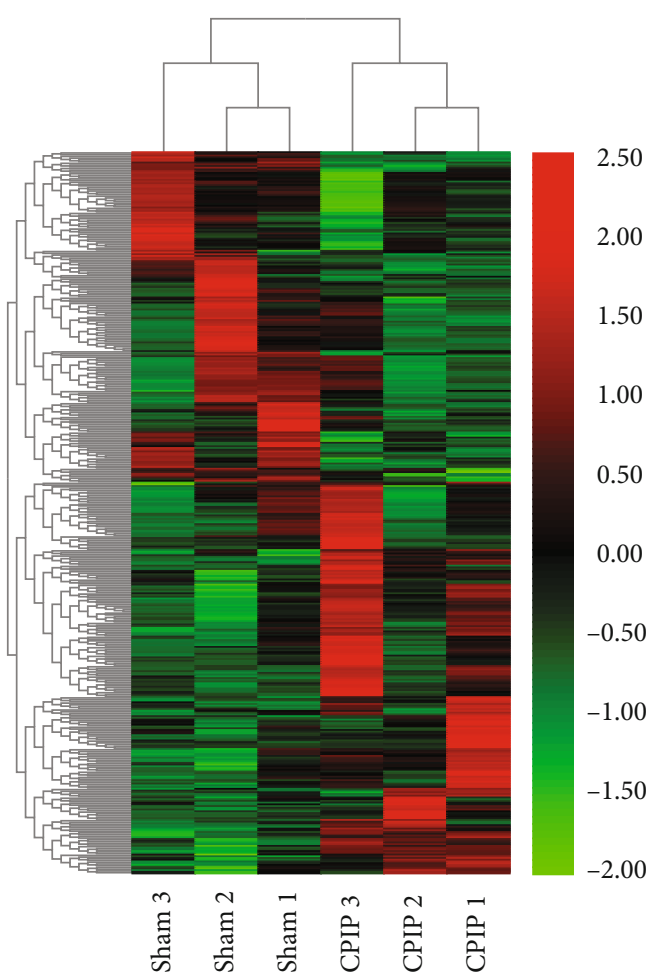

(a)

Biological process

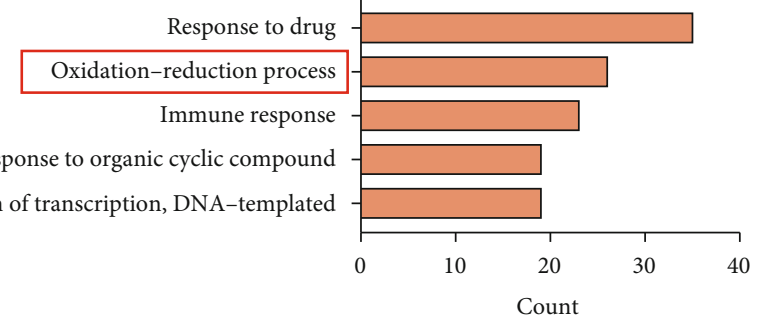

Cellular component
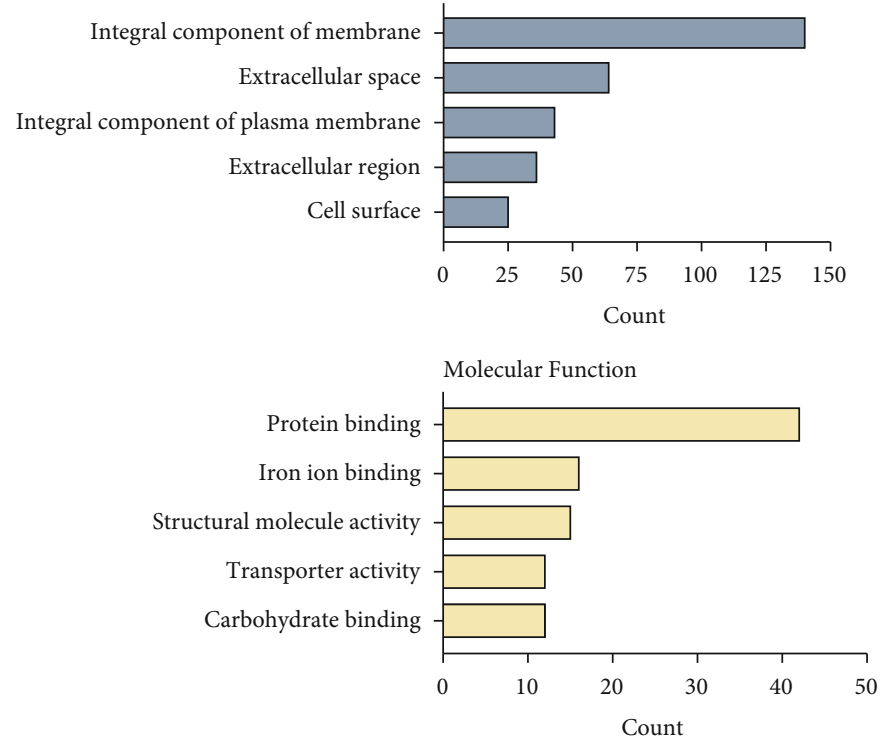

(b)

FIGURE 2: Continued. 


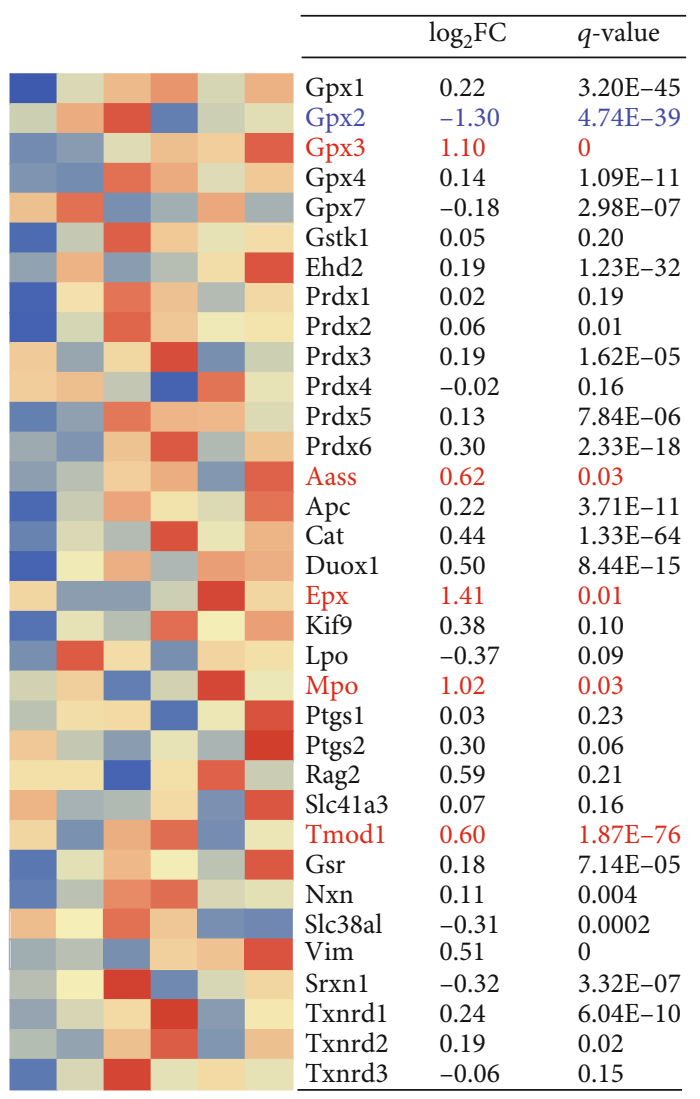

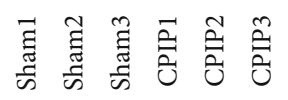

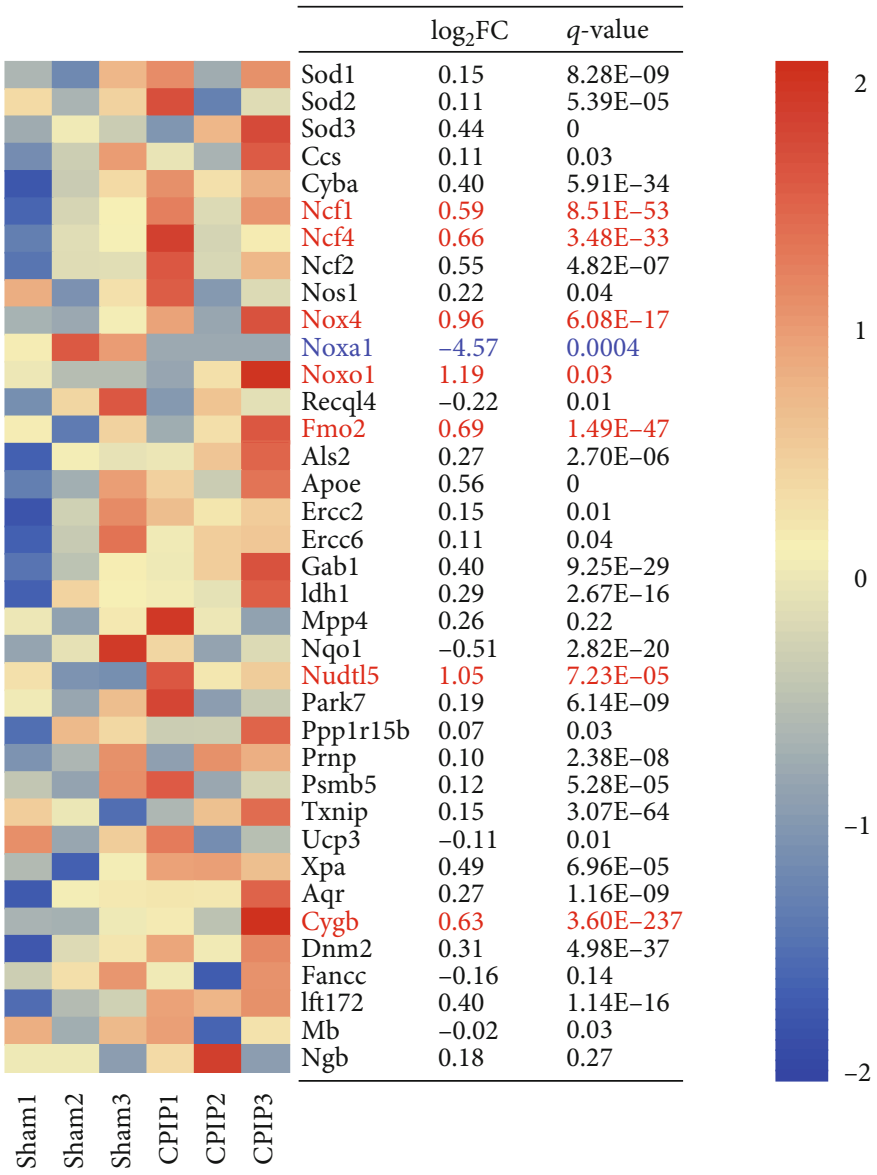

(c)

FIGURE 2: RNA-Seq profiling of gene expression in local ipsilateral hindpaw tissues of CPIP model rats. (a) Heat map showing hierarchical clustering patterns of DEGs from the control and CPIP model groups. (b) Gene Ontology (GO) pathway analysis of DEGs. The top 5 mostly enriched pathways were illustrated. (c) Heat map summarizing the genes particularly involved in oxidative stress, antioxidant defense, and reactive oxygen metabolism process as well as corresponding $\log _{2} \mathrm{FC}$ (FC: fold change) and $q$ value. Upregulated DEGs are displayed in red, whereas downregulated are in blue. Non-DEGs are in black. $n=3$ rats/group.

shows the top 5 most enriched pathways in each GO section. Of particular interest was "oxidation-reduction process," which ranked the $2^{\text {nd }}$ among all biological process. Oxidative stress is a factor that is considered to be involved triggering chronic pain [38]. We further chose some representative genes known to contribute to oxidative stress, antioxidant defense, and reactive oxygen metabolism [39] and evaluated the impact of CPIP model on these genes. Among the 71 genes tested, 14 were found to be significantly affected (Figure 2(b) and Suppl. Table 3), demonstrating oxidative stress may exist in local hindpaw tissues of CPIP rats.

\subsection{Oxidative Stress Occurred Primarily in Local Hindpaw} Tissues of CPIP Rats and Contributes to Mechanical Allodynia. We continued to study the oxidative stress in hindpaw tissues of male CPIP rats in more detail. We evaluated the changes of SOD and GSH-Px enzyme activities, two antioxidant enzymes. We also checked the contents of 4hydroxynonenal (4-HNE) and malondialdehyde (MDA), two lipid peroxidation products in ipsilateral hindpaw tissues. As shown in Figures 3(a) and 3(b), we found that
SOD and GSH levels were significantly decreased at days 3 and 7 , whereas the content of MDA was significantly increased through day 10 (Figure 3(c)). As shown in Figures 3(d) and 3(e), the content of 4-HNE was significantly increased at day 7 among CPIP rats. We proceeded to evaluate the oxidative stress status in spinal cord tissue and serum of CPIP model rats. SOD, GSH, and MDA levels were not significantly changed at day 7 , and 4-HNE level remained unchanged through day 14 in the ipsilateral spinal cord of CPIP model rats (Figures $3(f)-3(j))$. In addition, the immunoactivity of 8-OHG, a marker of oxidative damage in cellular nucleic acids, remains unchanged in the spinal cord during observation (till day 10, Suppl. Fig. 3A-C). Similarly, $\mathrm{SOD}, \mathrm{GSH}$, and MDA levels remained unchanged through day 10 in CPIP rat serum (Figures $3(\mathrm{k})-3(\mathrm{~m})$ ). Therefore, these data suggest that the oxidative stress occurred primarily in local hindpaw tissues of CPIP rats.

We then tested if the oxidative stress contributes to pain mechanisms of the rat model of CRPS-I. CPIP rats were administered with antioxidant NAC on a daily basis $(200 \mathrm{mg} / \mathrm{kg}$, i.p., Figure 4(a)). NAC administration 


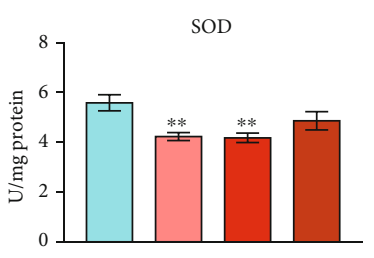

(a)

Ipsilateral hind paw tissues
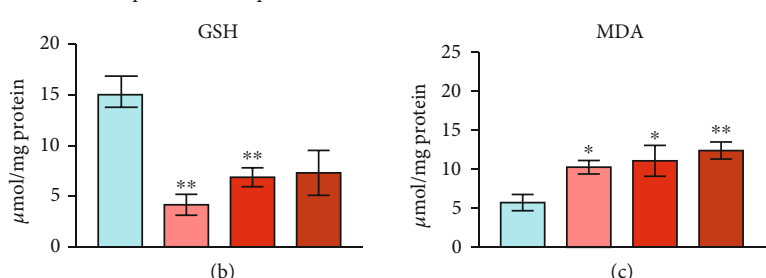

(c)
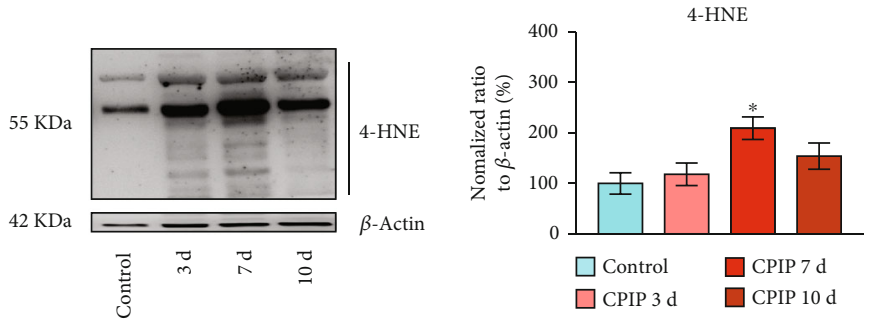

(d)

(e)

Ipsilateral spinal cord tissues
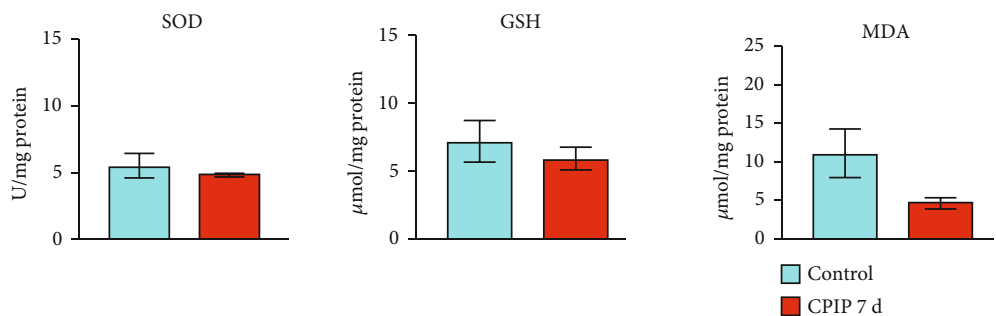

(f)

(g)

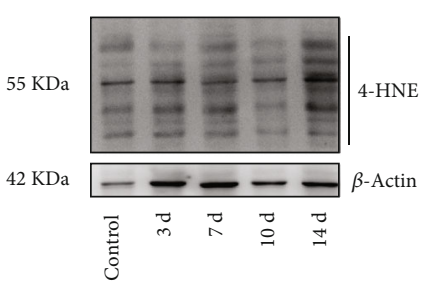

(i)

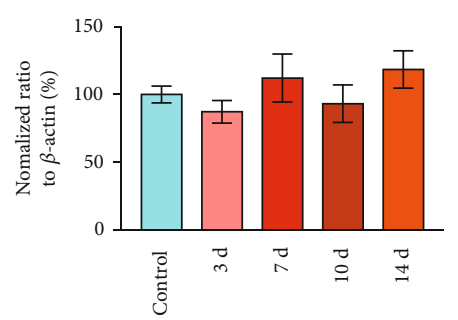

(j)

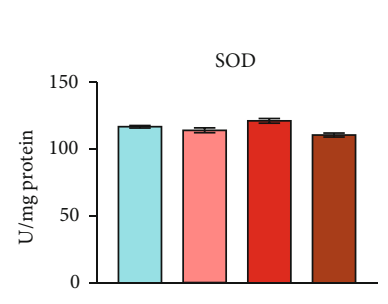

(k)

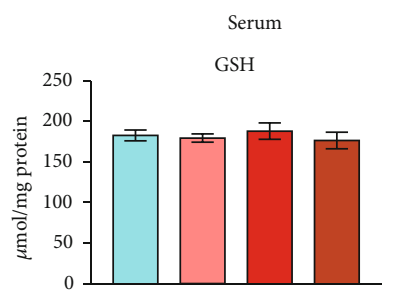

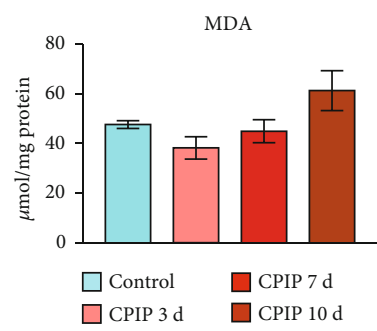

(m)

FIgURE 3: Evaluating oxidative stress status in ipsilateral hindpaw, the spinal cord, and serum of CPIP rats. (a-c) Results showing SOD activity (a), GSH-Px activity (b), and MDA content (c) determined in ipsilateral hindpaw tissues from control and CPIP model rats 3, 7, and $10 \mathrm{~d}$ after model establishment. (d) 4-HNE expression in ipsilateral hindpaw tissues determined by Western blotting at time points as indicated. Upper panel shows representative images of 4-HNE and lower panel shows $\beta$-actin. (e) Summarized results of 4-HNE expression normalized to $\beta$-actin. Results showing SOD activity (f), GSH-Px activity (g), and MDA content (h) determined in ipsilateral spinal cord tissues from control and CPIP model rats $7 \mathrm{~d}$ after model establishment. (i) 4-HNE expression in ipsilateral spinal cord tissues determined by Western blotting at time points as indicated. The upper panel shows representative images of 4-HNE, and the lower panel shows $\beta$-actin. (j) Summarized results of 4-HNE expression normalized to $\beta$-actin. Results showing SOD activity (k), GSHPx activity (l), and MDA content $(\mathrm{m})$ determined in serum from control and CPIP model rats 3, 7, and $10 \mathrm{~d}$ after model establishment. $n=5-6$ rats/group. 


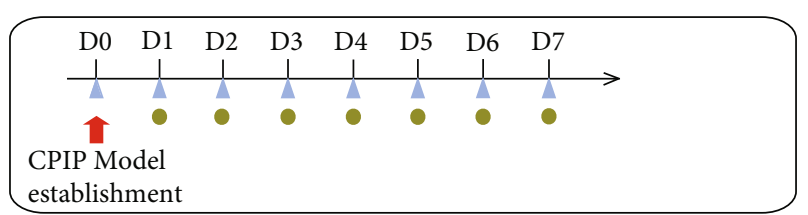

- Drug treatment

$\triangle$ Behavior test

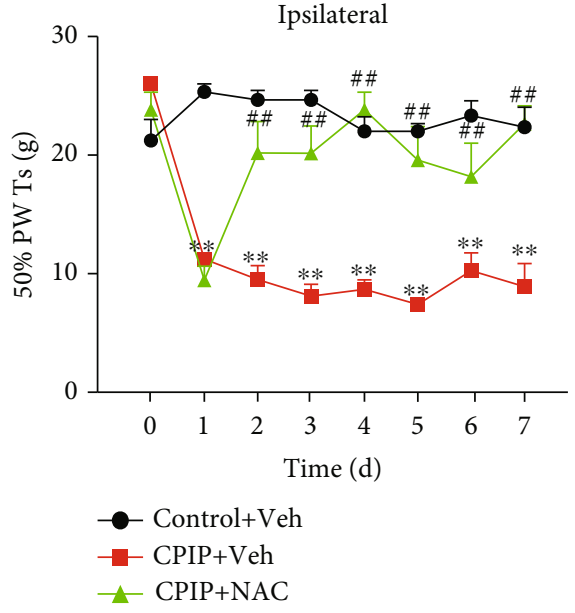

(b)

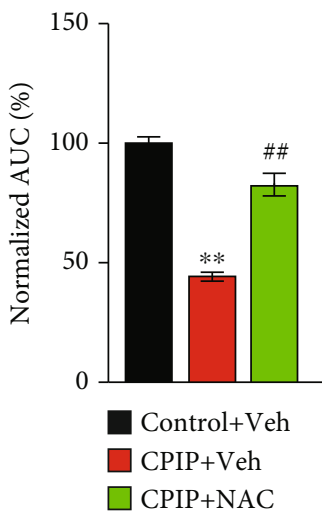

(c)

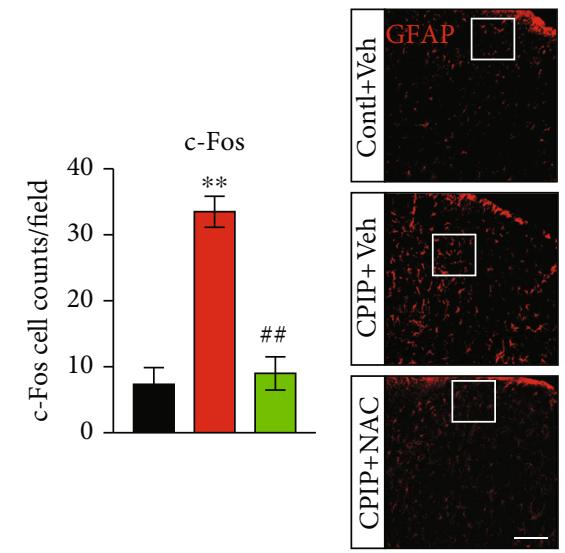

(e)
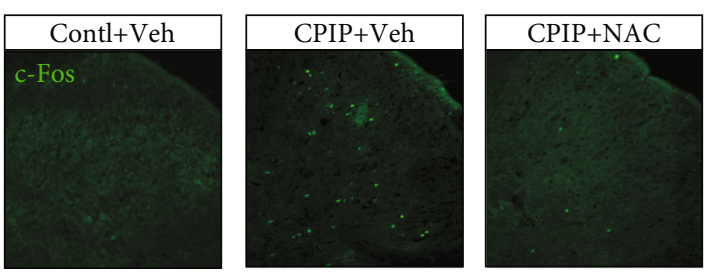

(d)

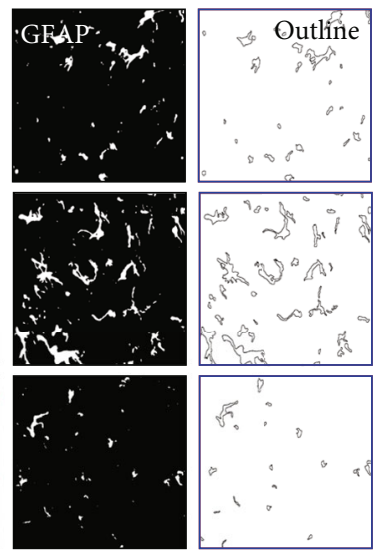

(f)

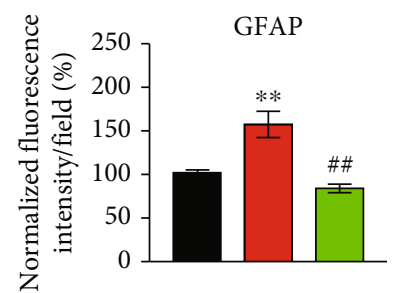

(g)

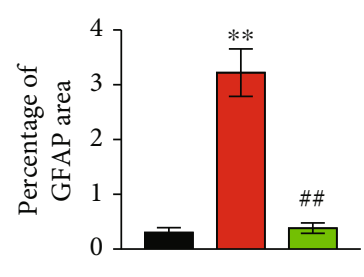

(h)

Figure 4: Continued. 

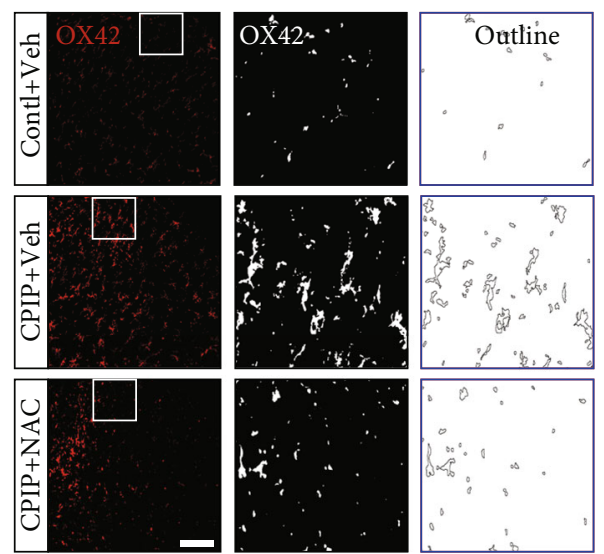

(i)

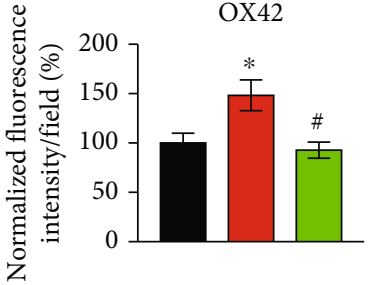

(j)

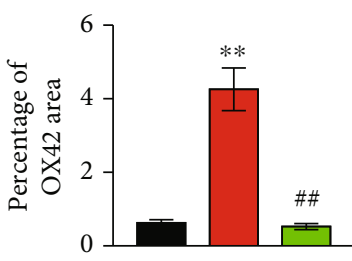

$(\mathrm{k})$

Figure 4: NAC treatment ameliorates mechanical allodynia of CPIP rats. (a) Schematic protocol showing time points for model establishment and NAC/vehicle treatment. (b) Time course of 50\% PWT changes after NAC/vehicle treatment. The CPIP+NAC group receives daily NAC $(200 \mathrm{mg} / \mathrm{kg}$, i.p.) treatment, whereas the CPIP+Veh group receives vehicle (PBS, i.p.) treatment. (c) Summary of AUC as in (b). (d) Ipsilateral $\mathrm{SCDH}$ with c-Fos staining from the control+Veh, CPIP+Veh, and CPIP+NAC groups. (e) Summary of the number of c-Fos-positive cells per observation field. (f) Ipsilateral SCDH with GFAP staining. Summary of the normalized fluorescence intensity (\%) of GFAP (g) and percentage of GFAP-stained area (h). (i) Ipsilateral SCDH with OX42 staining. Summary of the normalized fluorescence intensity (\%) of OX42 $(\mathrm{j})$ and percentage of OX42-stained area $(\mathrm{k})$. Scale bar $=100 \mu \mathrm{m} . n=5-6$ rats/group. ${ }^{*} p<0.05$ and ${ }^{* *} p<0.01 \mathrm{vs}$. the control+Veh group. ${ }^{\#} p<0.05$ and ${ }^{\# \#} p<0.01$ vs. the CPIP+Veh group.

effectively reduced the mechanical allodynia of ipsilateral hindpaw (Figure 4(b)). AUC analysis demonstrated an accumulated alleviation of mechanical allodynia in the CPIP+NAC group vs. CPIP+Veh group (Figure 4(c)). We then checked the effects of NAC treatment on spinal glial and neuron activation, which are critical processes contributing to central pain sensitization during chronic pain [40, 41]. Immunostaining experiments unraveled that c-Fos-labeled cell number was markedly increased in ipsilateral spinal cord dorsal horn $(\mathrm{SCDH})$ of the CPIP+Veh group vs. control+Veh group (Figures 4(d) and 4(e)). In addition, the immunostaining intensity of two markers for microglia and astrocyte, namely, OX42 and GFAP, was markedly increased in ipsilateral dorsal horn of the CPIP+Veh group vs. control+Veh group as well (Figures $4(\mathrm{f})-4(\mathrm{k})$ ). More importantly, the magnitudes of these cellular events were all reduced with NAC treatment (Figures $4(\mathrm{~d})-4(\mathrm{k})$ ).

We then tested whether locally increasing oxidative stress could mimic the chronic pain response of CPIP model rats. Daily local injection of $\mathrm{H}_{2} \mathrm{O}_{2}$ (an endogenous ROS product, $100 \mathrm{nmol} / \mathrm{site}$ ), into the hindpaws of naïve rats, elicited persistent mechanical allodynia (Figures 5(a) and 5(b)). AUC analysis indicated an accumulated development of mechanical allodynia in animals treated with $\mathrm{H}_{2} \mathrm{O}_{2}$ vs. vehicle (Figure 5(c)). In addition, immunostaining experiments indicated that persistent $\mathrm{H}_{2} \mathrm{O}_{2}$ injection produced c-Fos acti- vation (Figures 5(d) and 5(e)), as well as GFAP and OX42 overactivation (Figures 5(f)-5(k)) in ipsilateral SCDH of treated rats. This suggests that persistent local oxidative stress per se can cause mechanical allodynia, spinal neuron, and glial cell activation, which mimics chronic pain and spinal cellular events of CPIP model rats.

Sex-dependent pain mechanisms have been reported in certain pain conditions. We then evaluated the oxidative status in female CPIP model rats. As shown in Suppl. Fig. 4A$\mathrm{D}$, we found that SOD and GSH levels were significantly decreased, whereas $\mathrm{H}_{2} \mathrm{O}_{2}$ and MDA level was significantly increased in ipsilateral hindpaw tissues at day 7 in female CPIP model rats. By contrast, the contents of these substances were not obviously changed from the ipsilateral spinal cord (Suppl. Fig. 4E-H). Similar to male rats, NAC effectively ameliorated the mechanical hypersensitivity of ipsilateral hindpaws from female rats (Suppl. Fig. 4I). This result suggests that local oxidative stress can drive pain response in CPIP model animals in both sexes. In all, the above results demonstrate a critical role of local oxidative stress in hindpaw tissue that contributes to chronic pain mechanisms of a rat model of CRPS-I.

3.3. Electroacupuncture Alleviates Mechanical Allodynia of CPIP Rats through Reducing Local Oxidative Stress and Inflammation in Hindpaw Tissues. Electroacupuncture 

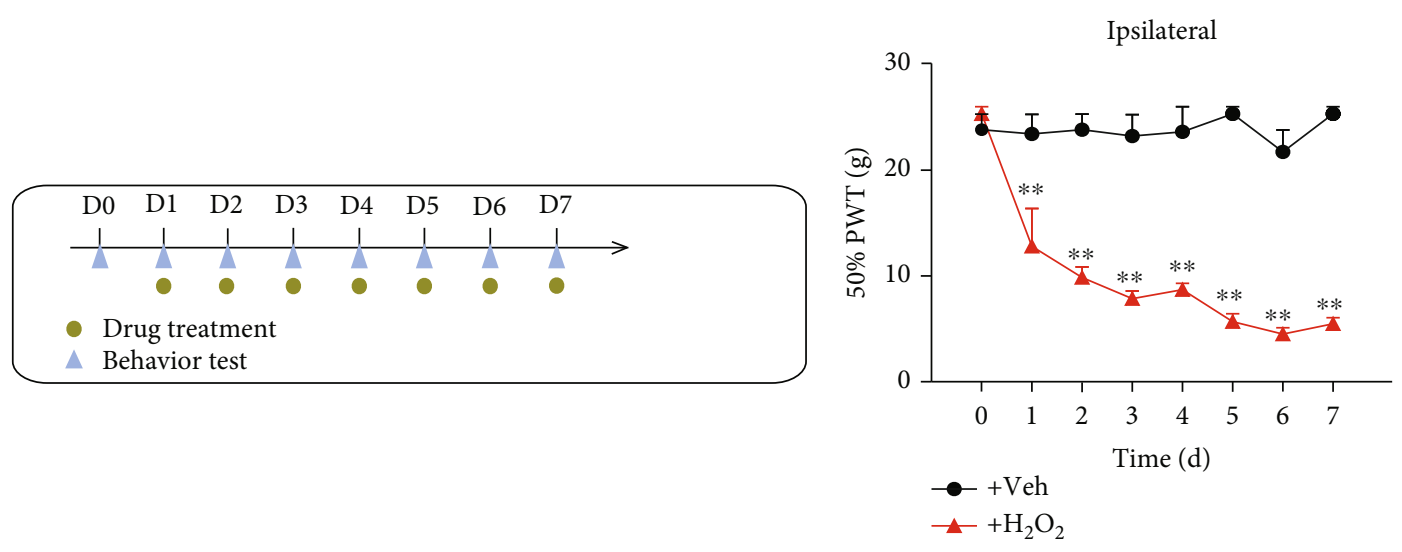

(a)

(b)

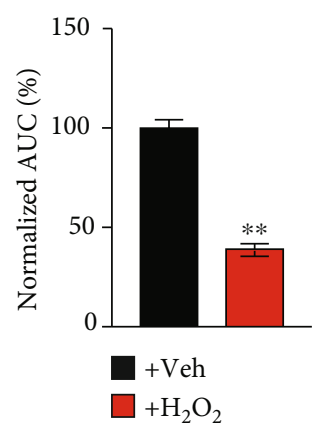

(c)

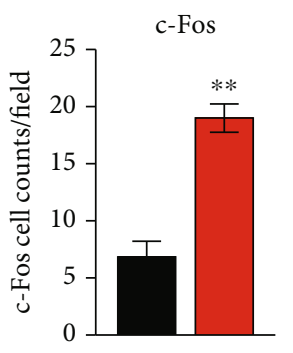

(e)
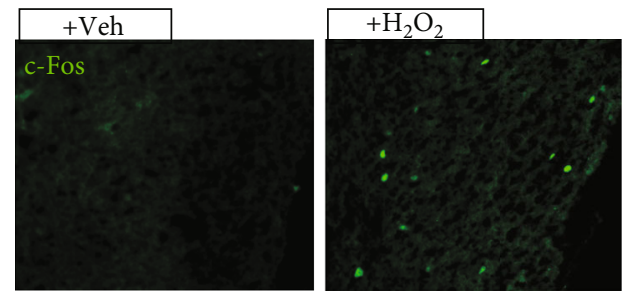

(d)
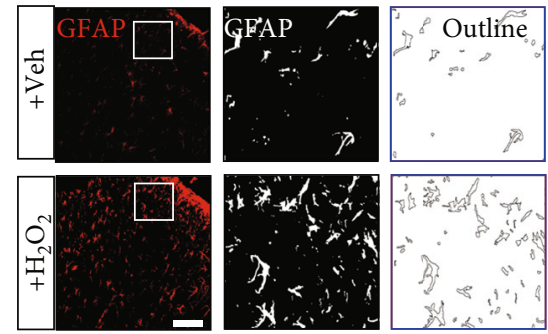

(f)

GFAP

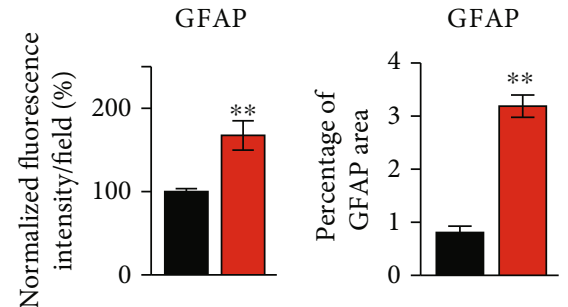

(h)
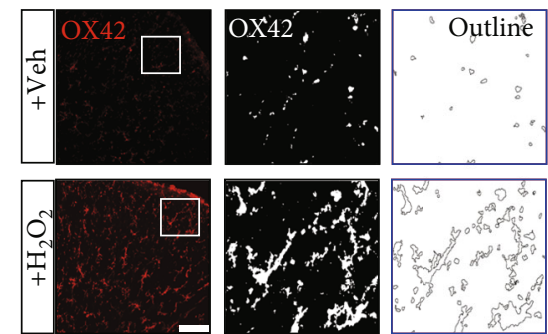

(i)

FIgURE 5: Continued. 


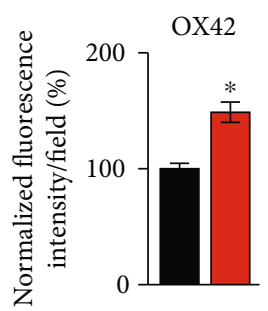

(j)

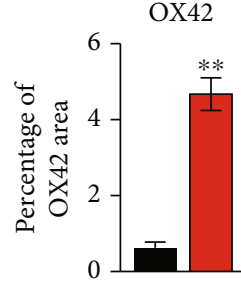

(k)

FIGURE 5: Locally increasing oxidative stress produces mechanical allodynia among naïve rats. (a) Schematic protocol showing time points for $\mathrm{H}_{2} \mathrm{O}_{2}$ /vehicle treatment in naïve rats. (b) Time course of $50 \%$ PWT changes after $\mathrm{H}_{2} \mathrm{O}_{2}$ /vehicle treatment. The $+\mathrm{H}_{2} \mathrm{O}_{2}$ group receives daily $\mathrm{H}_{2} \mathrm{O}_{2}(100 \mathrm{nmol} / \mathrm{site})$ injection into hindpaws, whereas the $+\mathrm{Veh}$ group receives only vehicle (PBS) injection. (c) Summary of AUC as in (b). (d) Ipsilateral SCDH with c-Fos staining from the + Veh vs. $+\mathrm{H}_{2} \mathrm{O}_{2}$ group. (e) Summary of the number of c-Fos-positive cells per observation field. (f) Ipsilateral SCDH with GFAP staining. Summary of the normalized fluorescence intensity (\%) of GFAP (g) and percentage of GFAP-stained area (h). (i) Ipsilateral SCDH with OX42 staining. Summary of the normalized fluorescence intensity of OX42 (j) and percentage of OX42-stained area (k). $n=6$ rats/group. ${ }^{* *} p<0.01$ vs. +Veh group.

(EA), a modified acupuncture therapy, is effective for pain management without obvious adverse effects [42]. EA showed positive effects on alleviating pain, improving limb dysfunction, and improving daily activities of CRPS-I patients [18]. Our recent study further demonstrated EA effectively alleviated mechanical allodynia of CPIP model rats and identified $2 / 100 \mathrm{~Hz}$ as an optimal therapeutic frequency [10]. However, the analgesic effects of EA on CRPS-I still remain elusive. Therefore, we employed 2/ $100 \mathrm{~Hz}$ EA on ipsilateral acupoints of BL60 and ST36 from CPIP model rats as we did in our recent study (Figure 6(a)) [10]. We found that daily EA treatment (for successive 7 days) effectively ameliorated bilateral mechanical pain hypersensitivities of CPIP rats (Figures 6(b)-6(e)), whereas sham EA had no effect, a result consistent with our recent study [10]. We proceeded to examine if the antiallodynic effect exerted by EA was possibly attributed to the modulation of local oxidative stress in the hindpaw. Figure 6(a) shows the experimental scheme and the time points for observation and tissue collection. Oxidative stress evaluation indicated that SOD and GSH levels were decreased significantly, whereas MDA and $\mathrm{H}_{2} \mathrm{O}_{2}$ levels were increased significantly in ipsilateral hindpaw tissues from model rats vs. control rats (Figures $7(a)-7(d)$ ). EA intervention significantly increased the SOD and GSH levels, while it decreased MDA and $\mathrm{H}_{2} \mathrm{O}_{2}$ overproductions vs. the sham EA group (Figures 7(a)-7(d)). 8-OHG immunoactivity was significantly increased in local hindpaw tissues from CPIP rats, and the intervention of EA significantly alleviated its overexpression (Figures 7(e) and 7(f)). Immunoblot experiments showed that 4-HNE overproduction from hindpaw tissues of CPIP rats was significantly attenuated by EA intervention, whereas sham EA had no such effect (Figure 7(g)).

Our study suggests that persistent local oxidative stress triggers the activation of glial cells in SCDH. We then continued to investigate if EA might attenuate these cellular events in the spinal cord, which constitutes critical process leading to central pain sensitization [43]. GFAP and OX42 immunoreactivity in SCDH rose markedly from model group vs. controls (Suppl. Fig. 5A-F). Electroacupuncture effectively reduced GFAP and OX42 immunoreactivity increase in ipsilateral SCDH (Suppl. Fig. 5A-F).
We then investigated how EA may modulate local oxidative stress in CPIP model rats. Nrf2 is a nuclear transcription factor that couples with antioxidant response element and coordinates with the activation of antioxidative genes and pathways [44]. Next, to investigate if EA may produce antioxidative effects through Nrf2 activation, we first examined Nrf2 expression. As shown in Figures 8(a) and 8(b), the protein level of Nrf2 was markedly reduced in hindpaw tissues of CPIP rats vs. the control group, whereas EA treatment restored Nrf2 expression. Antioxidant NAC produced similar effects on Nrf2 as EA (Figures 8(a) and 8(b)). Local application of Nrf2-specific antagonist ML385 abolished the antioxidative effects of EA by reducing GSH and SOD levels and increasing $\mathrm{H}_{2} \mathrm{O}_{2}$ and MDA levels in hindpaw tissues (Figures 8(c)-8(f)). Furthermore, ML385 treatment effectively abolished EA-mediated antiallodynia in CPIP rats (Figures $8(\mathrm{~g})$ and $8(\mathrm{~h})$ ). Taken together, these findings indicate that EA alleviates mechanical pain response of CPIP rats by reducing local oxidative stress via Nrf2-dependent antioxidative mechanism.

3.4. EA Reduced the Upregulation of Inflammatory Cytokines in Hindpaw Tissues and Reduced the Enhanced TRPA1 Channel Activity in Peripheral Sensory Neurons Innervating Hindpaw of CPIP Rat. Oxidative stress triggers the activation of a variety of transcription factors, resulting in upregulation of an array of inflammatory mediators [45]. We found that the gene expressions of some typical inflammatory cytokines, $T n f a, I l 1 b$, and $I l 6$, were significantly upregulated in ipsilateral hindpaw tissues of CPIP model rats. The expressions of these inflammatory genes were all reduced by NAC (Suppl. Fig. 6A-C), indicating an important role of oxidative stress in mediating the upregulation of these inflammatory genes. In addition, EA treatment produced similar reduction of Tnfa and $I l 1 b$ overexpression as NAC (Suppl. Fig. 6A\&B).

Many endogenous ROS molecules and lipid peroxidation products can target against neuronal TRPA1 channel in peripheral sensory neurons to produce nociception. IL$1 \beta$ and TNF- $\alpha$ may contribute to pain sensitization via promoting TRPA1 channel expression [46, 47]. We then set to determine and compare the functions of TRPA1 channel 

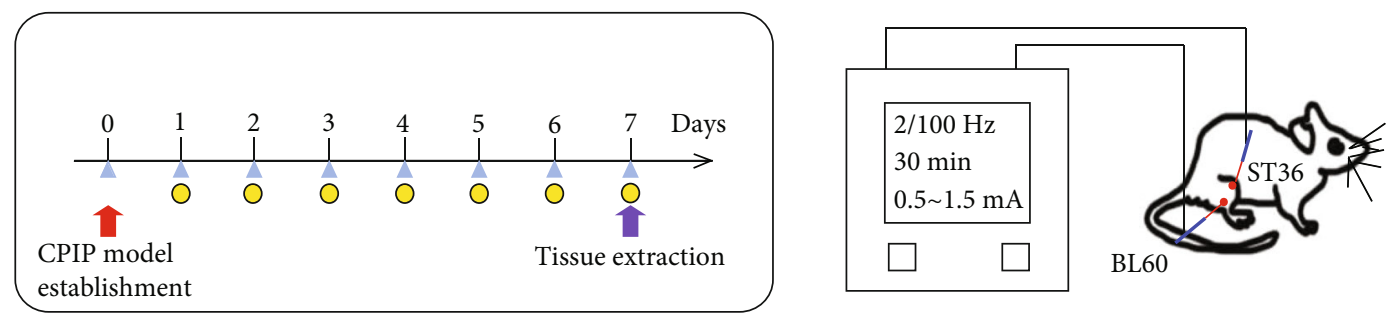

O EA/Sham EA

$\triangle$ Behavior test

(a)

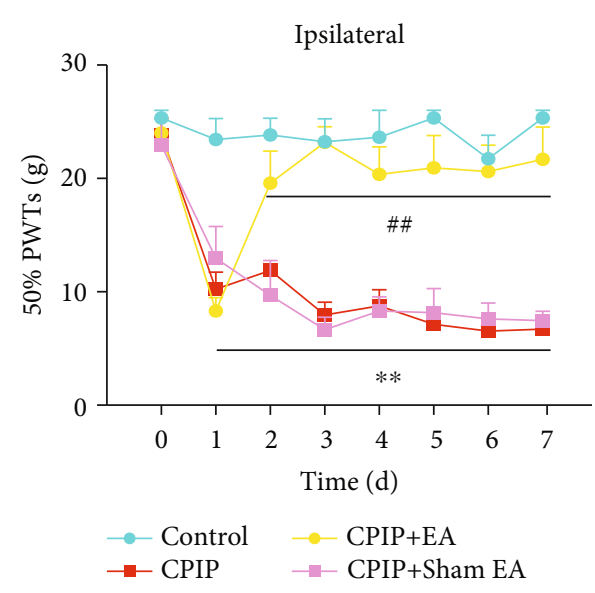

(b)

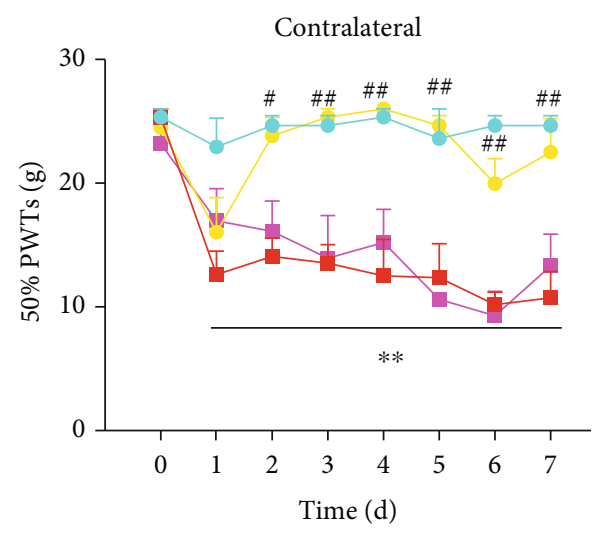

(d)

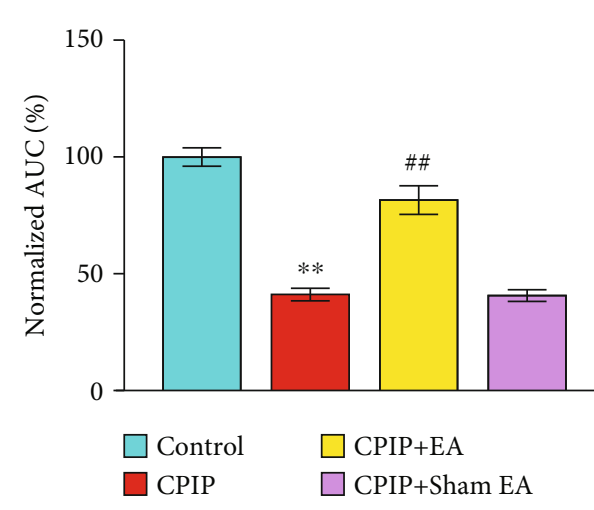

(c)

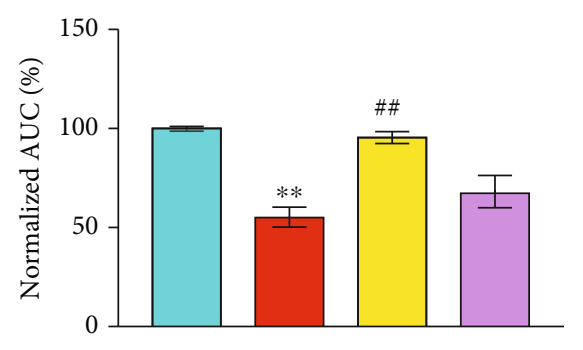

(e)

FIGURE 6: EA intervention ameliorates mechanical allodynia of CPIP rats. (a) Schematic protocol illustrating time points for EA or sham EA treatment in CPIP model rats (left) and schematic picture showing EA parameters and the locations of ST36 and BL60 acupoints on the rat (right). (b) Time course of the effect of EA/sham EA treatment on 50\% PWT of ipsilateral hindpaws of CPIP model rats. (c) Summary of AUC as in (b). (d) Time course of the effect of EA/sham EA treatment on 50\% PWT of contralateral hindpaws. (e) Summary of AUC as in (d). $n=6$ rats/group. ${ }^{* *} p<0.01$ vs. the control group. ${ }^{\# \#} p<0.01$ vs. the CPIP+sham EA group.

from peripheral dorsal root ganglion (DRG) neurons under CPIP and control conditions. Ipsilateral L4-6 DRG neurons innervating the hind limbs were acutely dissociated from CPIP model and control rats. The cells were then incubated with Fura-2AM and subject to imaging of intracellular $\left[\mathrm{Ca}^{2+}\right]$. We found that CPIP treatment significantly increased the proportion of $\mathrm{H}_{2} \mathrm{O}_{2}$-responsive neurons among all neurons, as well as the magnitude of $\mathrm{Ca}^{2+}$ responses to $\mathrm{H}_{2} \mathrm{O}_{2}$ (Figures 9(a)-9(d)). EA intervention largely decreased the proportion of $\mathrm{H}_{2} \mathrm{O}_{2}$-responsive neu- rons as well as the magnitude of $\mathrm{Ca}^{2+}$ responses compared with sham EA (Figures 9(a)-9(d)). Meanwhile, NAC treatment produced similar effects as EA (Figures 9(a)-9(d)). These results suggest that the function of neuronal TRPA1 was enhanced under CPIP condition in a ROS-dependent manner, whereas EA intervention reverses the enhanced TRPA1 channel activities.

3.5. EA Improved Regional Blood Flow Dysfunction in Affected Hindpaws of CPIP Model Rats. CRPS-I patients 
Ipsilateral hind paw tissues

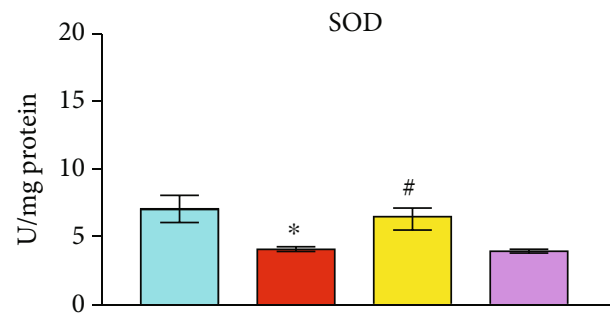

(a)

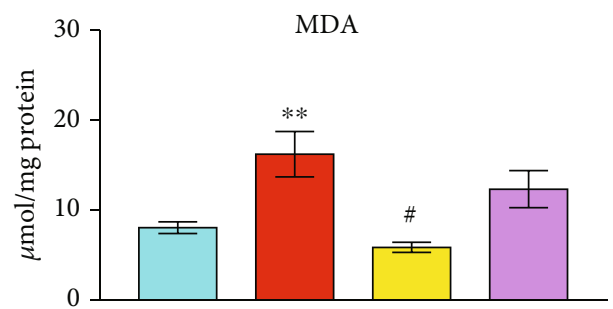

(c)

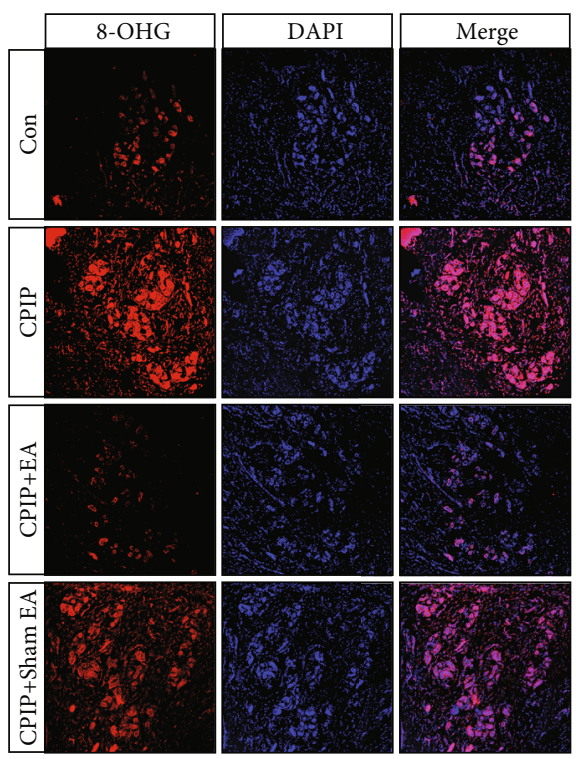

(e)

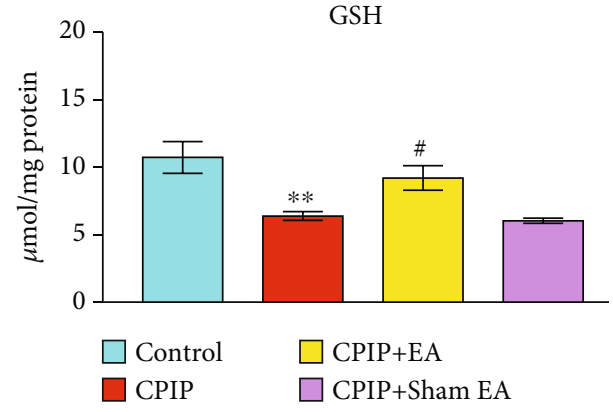

(b)

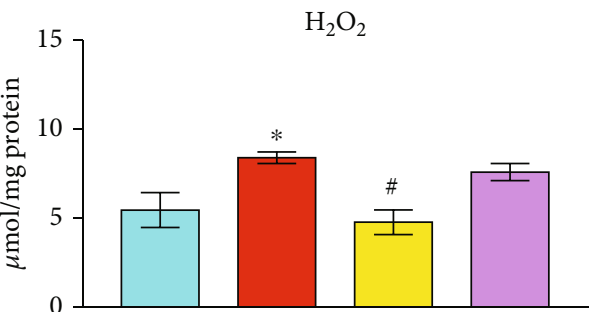

(d)

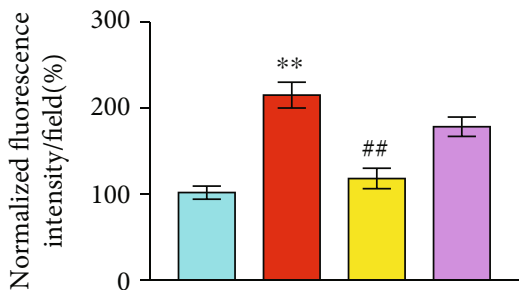

(f)
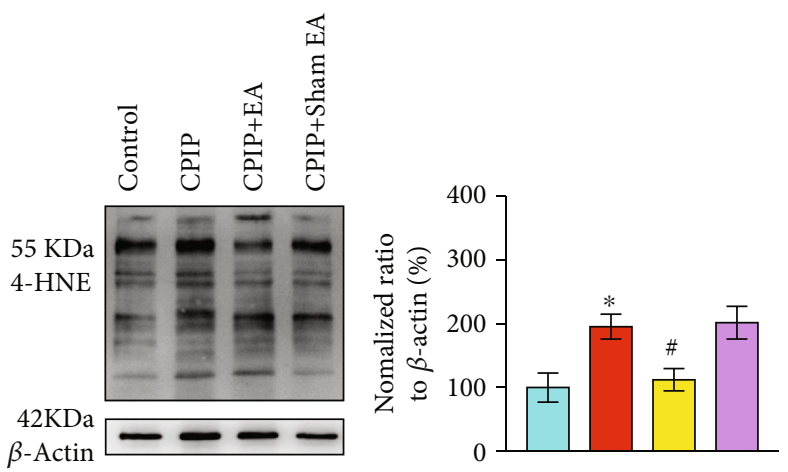

$(\mathrm{g})$

FIgURE 7: EA intervention ameliorates excessive oxidative stress in local hindpaw tissues of CPIP rats. Results showing SOD activity (a), GSH-Px activity (b), MDA content (c), and $\mathrm{H}_{2} \mathrm{O}_{2}$ content (d) determined in ipsilateral hindpaw tissues of the control, CPIP, CPIP+EA, and CPIP+sham EA groups $7 \mathrm{~d}$ after model establishment. (e) Representative pictures showing 8-OHG immunostaining in ipsilateral hindpaw tissues of each group. (f) Summarized data of normalized fluorescence intensity of 8-OHG per observation field of each group. (g) 4 -HNE expression determined by Western blot in ipsilateral hindpaw tissues of each group. $n=6$ rats/group. ${ }^{*} p<0.05$ and ${ }^{* *} p<0.01$ vs. the control group. ${ }^{\#} p<0.05$ and ${ }^{\# \#} p<0.01$ vs. the CPIP+sham EA group.

showed microvascular injury and decreased blood flow in affected limbs [48]. These symptoms are among the key clinical features of CRPS-I patients. We then monitored the regional blood flow from the affected hindpaws of CPIP rats using laser Doppler. Results showed that CPIP model rats exhibited significantly decreased regional blood flow in hindpaw $1 \mathrm{~d}$ after model establishment and persisted until day 7 (Figures 10(a) and 10(b)). EA treatment significantly improved regional blood flow in hindpaw (Figures 10(a) and $10(\mathrm{~b}))$. Moreover, NAC treatment produced similar 


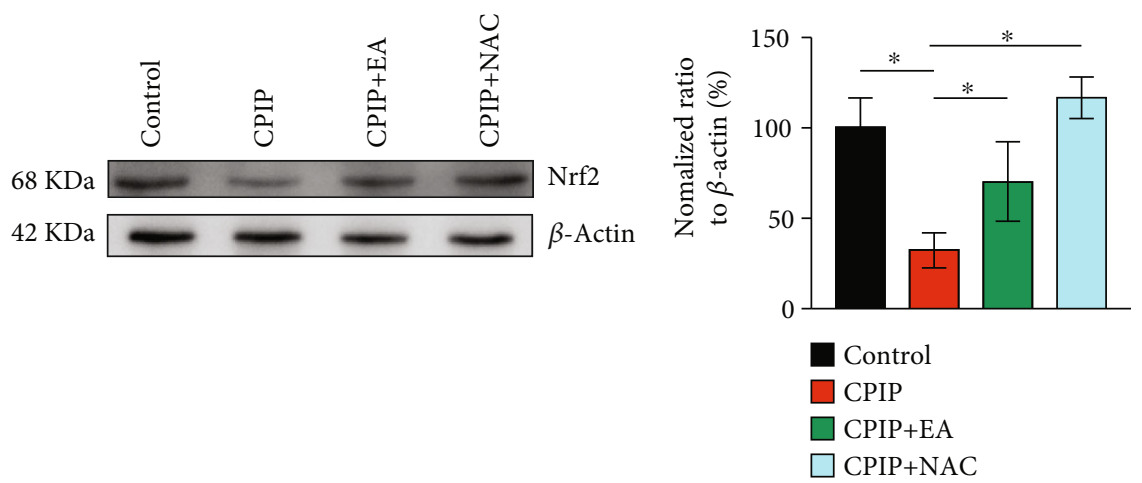

(a)

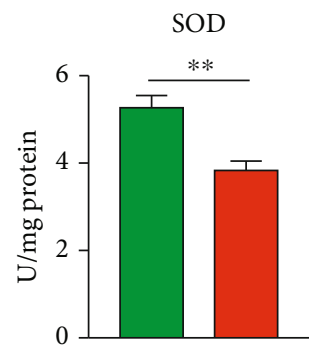

(c)

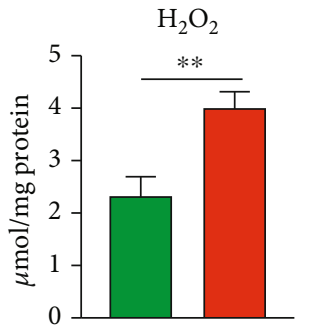

(e)

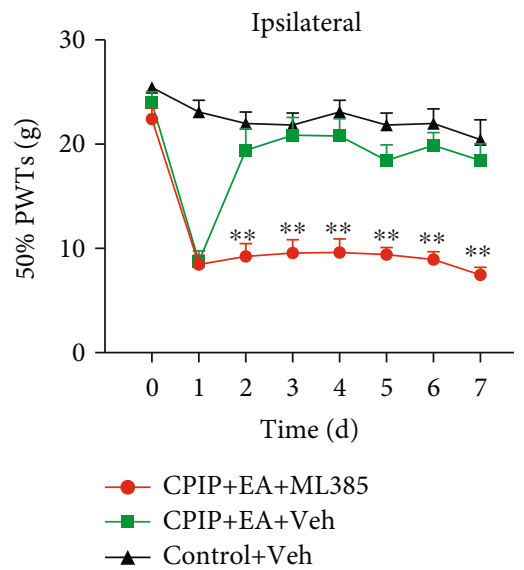

(g)

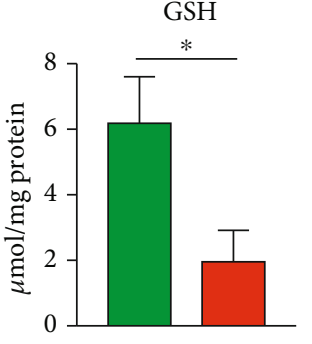

(d)

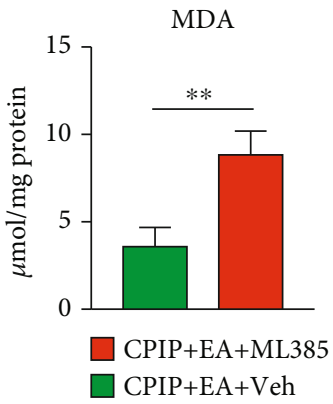

(f)

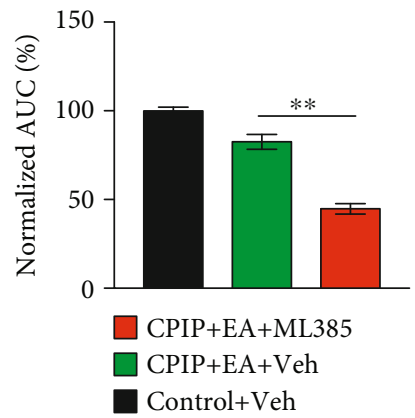

$(\mathrm{h})$

FIGURE 8: EA exerts antiallodynic effect on CPIP rats through activating Nrf2 in affected hindpaw. (a) Nrf2 expression determined by Western blotting in ipsilateral hindpaw tissues of the control, CPIP, CPIP+EA, and CPIP+NAC groups $7 \mathrm{~d}$ after model establishment. The upper panel shows representative images of $\mathrm{Nrf2}$, and the lower panel shows $\beta$-actin. (b) Summarized results of Nrf2 expression normalized to $\beta$-actin. Evaluation of SOD activity (c), GSH-Px activity (d), $\mathrm{H}_{2} \mathrm{O}_{2}$ (e), and MDA content (f) in ipsilateral hindpaw tissues from the CPIP+EA+ML385 and CPIP+EA+Veh group rats $7 \mathrm{~d}$ after model establishment. (g) Time course of the effect of intraplantar injecting Nrf2 antagonist ML385 (400 $\mu \mathrm{g} / \mathrm{rat})$ or corresponding vehicle (1\% DMSO in PBS) on 50\% PWT of ipsilateral hindpaws of CPIP model rats. (h) Summary of AUC as in (g). $n=6$ rats/group. ${ }^{*} p<0.05$ and ${ }^{* *} p<0.01$ vs. CPIP+EA+Veh or as indicated. 


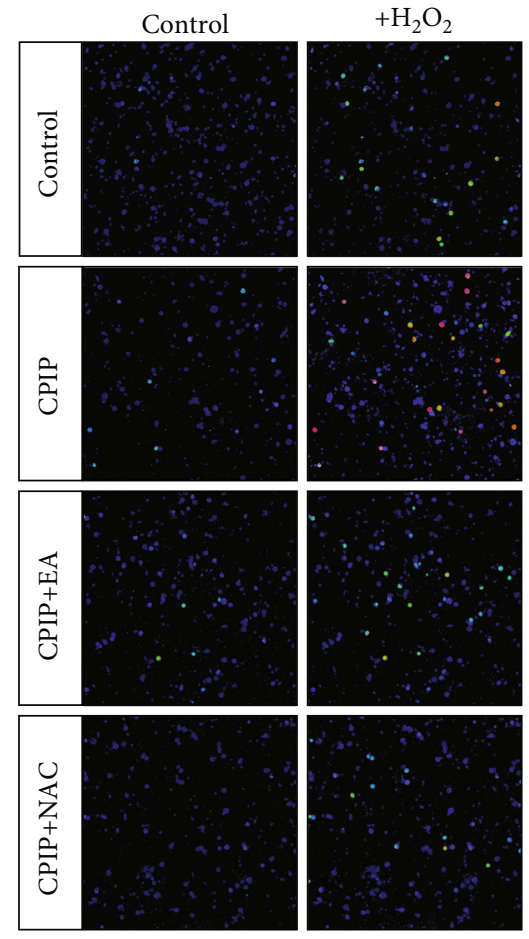

(a)

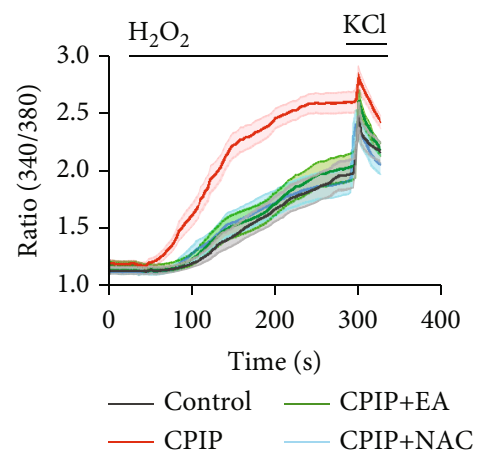

(c)

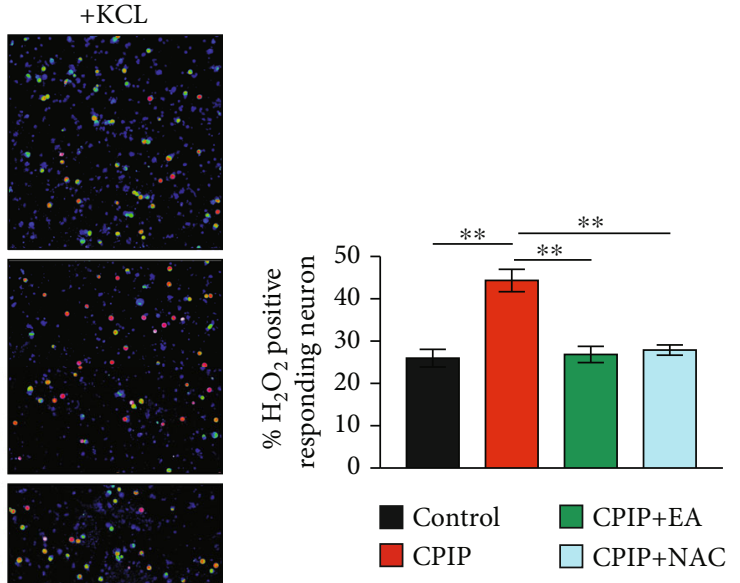

(b)

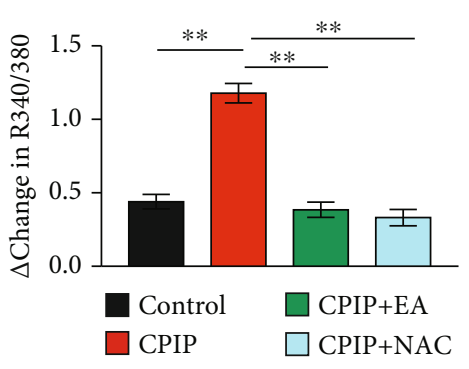

(d)

FIGURE 9: TRPA1 channel functional activity is enhanced in DRG neurons of CPIP rats, whereas repetitive EA or NAC treatment reversed TRPA1's enhanced activity. (a) Pseudocolor images from Fura-2-based ratiometric $\mathrm{Ca}^{2+}$ imaging. The pictures indicate $\mathrm{Ca}^{2+}$ responses from ipsilateral L4-6 DRG neurons derived from the control, CPIP, CPIP+EA, and CPIP+NAC group rats. $\mathrm{H}_{2} \mathrm{O}_{2}(500 \mu \mathrm{M})$, an endogenous TRPA1 agonist, was used to stimulate TRPA1 in these neurons. $\mathrm{KCl}(40 \mathrm{mM})$ was applied to the bath at the end of each experiment to identify all live neurons. (b) Summary of the percentage of $\mathrm{H}_{2} \mathrm{O}_{2}$ positively responding neurons in each observation field from all groups as mentioned above. $n=6-7$ tests/group. Each group contains 150-200 neurons derived from 3-4 rats. (c) Comparison of averaged Ca ${ }^{2+}$ responses triggered by $\mathrm{H}_{2} \mathrm{O}_{2}$ among all groups. $n>40$ cells/group. (d) Data summary showing $\Delta$ increase of peak $340 / 380$ ratio. $n>60$ neurons/group derived from 3-4 rats/group. ${ }^{* *} p<0.01$.

improvement in blood flow as EA (Figures 10(c) and 10(d)). This result implies that regional blood flow dysfunction of CPIP model rats is ROS dependent and EA improves the dysregulated regional blood flow.

\section{Discussion}

Tissue inflammation and oxidative injury are considered important contributing factors to CRPS-I pathogenesis [49]. Antioxidants have shown pain-relieving effects on animal models of CRPS-I [7, 50, 51]. However, studies that systematically evaluated oxidative stress status in CRPS-I are still lacking. It remains unknown where the oxidative stress is predominantly generated and affected. In this study, we systematically evaluated oxidative stress status in a rat CRPS-I model, from the periphery to the spinal cord level. We found that oxidative stress occurred primarily in local affected hindpaw tissues but not in the spinal cord or serum of CPIP rats. These observations are relevant to one study showing oxidative stress occurred in hindpaw tissues of CPIP rats [13]. Moreover, oxidative stress in plasma of CRPS patients is not elevated compared to matched healthy volunteers [52]. We further found that locally increasing oxidative stress by applying $\mathrm{H}_{2} \mathrm{O}_{2}$ is sufficient to induce chronic pain 


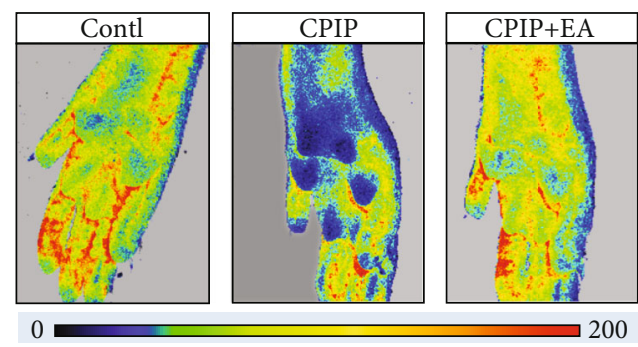

(a)

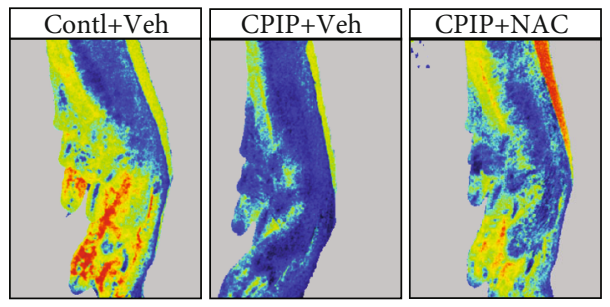

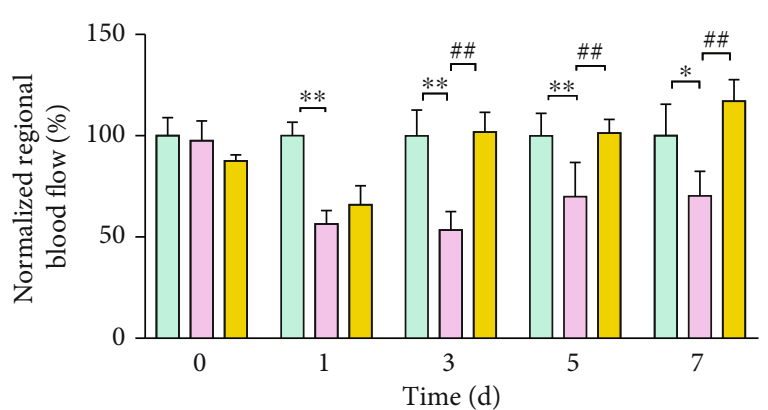

$\square$ Contl

$\square$ CPIP

$\square \mathrm{CPIP}+\mathrm{EA}$ (c)

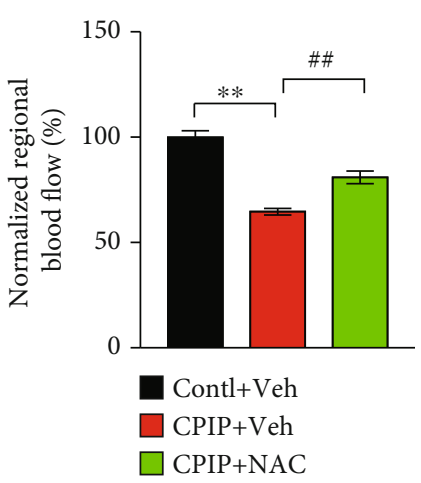

(d)

FIGURE 10: EA or NAC treatment improved regional blood flow dysfunction in affected hindpaws of CPIP model rats. (a) Representative pseudocolor images showing blood flow status in ipsilateral hindpaws of the control, CPIP, and CPIP+EA groups. (b) Time course summary of normalized blood flow of each group. Control group results were taken as 100\%, and results of all other groups were normalized accordingly. (c) Representative pseudocolor images showing blood flow status in ipsilateral hindpaw tissues of the control+Veh, CPIP+Veh, and CPIP+NAC groups. (d) Summary of normalized blood flow of each group at day 7 . ${ }^{*} p<0.05,{ }^{* *} p<0.01$, and ${ }^{\# \#} p<0.01 . n=5-7$ rats/group.

and spinal glial cell overactivation in naive rats. These results suggest that ROS production in local affected hindpaw is an important mechanism contributing to the mechanical hypersensitivities among CPIP rats. These findings imply that targeting local oxidative stress in affected extremities might be effective approaches for relieving CRPS-I.

Emerging evidence indicates sex-dimorphic mechanism exists in driving certain chronic pain $[53,54]$. Sex dimorphism in pain perception has gained increasing attention in both clinical and experimental studies. Epidemiology studies indicated females are more prone to develop CRPSI than males [55]. Therefore, we tested whether local oxidative stress-induced pain in CPIP model animals was driven in a sex-dimorphic manner. We found that female CPIP model rats exhibit similar magnitude of oxidative stress increase in local affected hindpaw as male rats. No upregulation of oxidative stress in the spinal cord was observed in female rats. Moreover, NAC treatment attenuated pain response of female model rats in similar scale as male model rats. Therefore, these data suggest that local oxidative stress can drive pain response in CPIP model animals regardless of sex differences.

TRPA1 channel is distributed in a subgroup of peptidergic nociceptive neurons and can trigger neurogenic inflam- mation as well as nociception upon its activation. TRPA1 is known to be activated by endogenous ROS as well as lipid peroxidation products, including $\mathrm{H}_{2} \mathrm{O}_{2}, 4-\mathrm{HNE}$, and OxPAPC [32, 56, 57]. 4-HNE has been identified to be increased in hindpaw tissues of CPIP model rats previously [13]. Our study further found that, in addition to 4-HNE, another ROS product $\mathrm{H}_{2} \mathrm{O}_{2}$ was increased in affected hindpaw tissues of CPIP model rats as well. Pharmacological blockage of TRPA1 has been shown to be effective for ameliorating pain in CPIP model animals [13]. Therefore, these two endogenous agonists of TRPA1 may work together to activate TRPA1 in vivo and contribute to pain mechanisms of CRPS-I. We further found that the functional TRPA1 channel activity was markedly upregulated in DRG neurons from CPIP rats. In addition, NAC or EA treatment significantly reduced the functional enhancement of TRPA1 channel. Oxidative stress triggers the activation of a variety of transcription factors, resulting in the upregulation of an array of inflammatory mediators. Specific inflammatory mediators, e.g., IL- $1 \beta$, TNF- $\alpha$, and IL-6, are capable of enhancing TRPA1 channel expression $[46,47]$. The expressions of these cytokines were all increased in affected hindpaw tissues of CPIP model rats, but reduced by NAC or EA treatment. Thus, the enhanced functional TRPA1 
channel activity in CPIP model rats may be attributed to ROS-dependent inflammatory cytokine release.

There are emerging evidences demonstrating the role of oxidative stress in chronic pain. ROS and lipid peroxidation products as a result of oxidative stress contribute to chronic pain via multiple mechanisms, including direct activation of nociceptor TRPA1 in the periphery and activation of microglia in the spinal cord, affecting spinal synaptic plasticity or producing pain-inducing substances [32, 58-60]. ROS scavengers or NADPH oxidase inhibitors, which reduce oxidative stress and ROS production, ameliorate chronic pain [61-64]. The Nrf2 antioxidant signaling is an endogenous machinery to counteract oxidative stress. Recently, several studies demonstrated that activating the Nrf2 antioxidant signaling exerts potent analgesic effects on chronic pain through alleviating ROS-associated pathological processes (e.g., inflammation and glial overactivation) and improving mitochondrial bioenergetics in the peripheral and/or central nervous system $[65,66]$. Thus, activating the Nrf2 antioxidant signaling is a promising antinociceptive approach [67].

EA is an effective therapy to relieve pain and improve life quality of CRPS-I patients [18]. Our recent study demonstrated EA effectively reduces mechanical pain hypersensitivities in CPIP rats [10]. Here, we continued to explore how EA exerts therapeutic effects on CRPS-I. We found that EA significantly alleviated local oxidative stress in local hindpaw of CPIP rats and promoted antioxidant transcription factor Nrf2 upregulation. It has been reported that EA can restore the decreased levels of SOD and enhance Nrf2 expression in DRG of paclitaxel-induced peripheral neuropathic pain model rats [68]. However, it remains unknown whether EA-induced upregulation of Nrf2 contributes to its antioxidative and analgesic effect. In this study, we addressed this important question by testing the effects of pharmacological blocking Nrf2. We found that pharmacological blocking Nrf2 attenuated EA-induced antioxidative effects as well as analgesic effects. Therefore, our study established a causal relationship between EA-induced Nrf2 upregulation and EA-induced analgesic effect and proposed that Nrf2-mediated antioxidative effect contributes to EA's antiallodynic effect.

CPIP rats displayed contralateral mechanical allodynia, a sign of mirror image pain (MIP). At present, the pathogenesis of MIP remains largely unknown. Both central and peripheral mechanisms have been proposed to contribute to MIP [69]. It is found that glial cell showed overactivation in contralateral SCDH of CPIP rats, which could contribute to MIP $[11,14]$. In addition, we recently found that contralateral DRG of CPIP rats showed multiple pain-related gene expression changes, indicating peripheral mechanisms may also contribute to MIP [70]. But how contralateral spinal glial cells and DRG are activated or affected during CPIP remains unknown. It is possible that the ipsilateral DRG or the spinal cord releases certain inflammatory mediators upon stimulation that can diffuse to the contralateral side via cerebrospinal fluid. Then, these inflammatory mediators may act upon contralateral spinal glial cells and DRG to produce MIP [71]. Since we also found that EA can ameliorate MIP of CPIP rats, thus, it will be tempting to explore whether EA could affect the abovementioned MIP mechanisms, both peripherally and centrally.

Tissue ischemia is a possible contributing factor for CRPS-I pathogenesis [72]. Ischemic tissue injury results in the production of ROS and inflammatory cytokines that can cause vascular disturbance and blood flow dysfunction [49]. Microvascular injury and blood flow dysfunction lead to tissue hypoxia and even more ROS production, all of which may contribute to pain $[49,73]$. Impaired microcirculation and regional blood flow dysfunction in distal part of the affected extremity are important clinical features of CRPS-I [74, 75]. Here, we observed CPIP rats exhibit obvious regional blood flow dysfunction in affected hind limbs by laser Doppler, mimicking clinical feature of CRPS-I patients. NAC treatment improved the blood flow dysfunction of CPIP model rats, demonstrating an important contribution of ROS to its pathogenesis. Moreover, we found that EA treatment improved the regional blood flow dysfunction of CPIP model rats in similar degree as NAC. Since EA can produce similar antioxidative effect as NAC, we thus propose that the improvement of blood flow dysfunction by EA could be related with its antioxidative effect.

\section{Conclusions}

In all, we performed a systemic investigation of the oxidative stress status in a rat model for CRPS-I. We further identified local oxidative stress in affected hindpaw tissues as an important factor contributing to pathogenesis of CPIP model animals. EA can target against local oxidative stress by enhancing endogenous Nrf2-mediated antioxidative mechanism to relieve pain as well as inflammation in CRPS-I model animals. This work suggests targeting Nrf2 may be an effective way for relieving CRPS-I and EA is a promising method for CRPS-I management in clinical practice.

\section{Data Availability}

The key data are contained in the figures, tables, and additional files. The datasets used and/or analyzed during this study can be further obtained from the corresponding authors on reasonable request.

\section{Disclosure}

Contents are solely the responsibility of the authors and do not necessarily represent the official views of the funders.

\section{Conflicts of Interest}

The authors declare that they have no known competing financial interests or personal relationships that could have appeared to influence the work reported in this paper.

\section{Authors' Contributions}

Xiaojie Li, Chengyu Yin, Qimiao Hu, and Jie Wang contributed equally to this work. 


\section{Acknowledgments}

We thank the Academy of Chinese Medicine Sciences, Zhejiang Chinese Medical University, for the technical support to our work. This project was supported by the National Natural Science Foundation of China (81873365, 82105014, and 81603676), the Zhejiang Provincial Natural Science Funds for Distinguished Young Scholars (LR17H270001), the Zhejiang Provincial Natural Science Funds (LQ21H270004), and the research funds from Zhejiang Chinese Medical University (2021JKZDZC07, Q2019J01, and KC201943).

\section{Supplementary Materials}

The supplementary materials contain the following figures and tables in one file: Suppl. Figure 1: original Western blot images. Suppl. Figure 2: high-quality hindpaw tissue RNA obtained for RNA-Seq. Suppl. Figure 3: oxidative stressinduced cellular damage is not present in ipsilateral spinal cord dorsal horn of CPIP model rats. Suppl. Figure 4: evaluation of oxidative stress status in female CPIP model rats. Suppl. Figure 5: persistent EA treatment reduces overactivation of glial cells in SCDH of CPIP model rats. Suppl. Figure 6: persistent EA or NAC treatment reduces proinflammatory cytokine overexpression in hindpaw tissues of CPIP model rats. Suppl. Table 1: sequence of primers used for qPCR. Suppl. Table 2: complete list of statistical results (mean, SEM, SD, and confidence interval). Suppl. Table 3: expression changes of genes involved in oxidative stress, antioxidant defense, and reactive oxygen metabolism process. (Supplementary Materials)

\section{References}

[1] S. Ott and C. Maihofner, "Signs and symptoms in 1,043 patients with complex regional pain syndrome," The Journal of Pain, vol. 19, pp. 599-611, 2018.

[2] F. Birklein, S. K. Ajit, A. Goebel, R. Perez, and C. Sommer, "Complex regional pain syndrome - phenotypic characteristics and potential biomarkers," Nature Reviews. Neurology, vol. 14, no. 5, pp. 272-284, 2018.

[3] I. Urits, A. H. Shen, M. R. Jones, O. Viswanath, and A. D. Kaye, "Complex regional pain syndrome, current concepts and treatment options," Current Pain and Headache Reports, vol. 22, p. 10, 2018.

[4] R. Freeman, R. Edwards, R. Baron et al., "AAPT diagnostic criteria for peripheral neuropathic pain: focal and segmental disorders," The Journal of Pain, vol. 20, pp. 369-393, 2019.

[5] H. Shim, J. Rose, S. Halle, and P. Shekane, "Complex regional pain syndrome: a narrative review for the practising clinician," British Journal of Anaesthesia, vol. 123, no. 2, pp. e424-e433, 2019.

[6] D. H. Lee, E. C. Noh, Y. C. Kim et al., "Risk factors for suicidal ideation among patients with complex regional pain syndrome," Psychiatry Investigation, vol. 11, pp. 32-38, 2014.

[7] T. J. Coderre, D. N. Xanthos, L. Francis, and G. J. Bennett, "Chronic post-ischemia pain (CPIP): a novel animal model of complex regional pain syndrome-type I (CRPS-I; reflex sympathetic dystrophy) produced by prolonged hindpaw ischemia and reperfusion in the rat," Pain, vol. 112, pp. $94-$ 105, 2004.

[8] Q. Hu, X. Zheng, R. Chen et al., "Chronic post-ischemia pain model for complex regional pain syndrome type-I in rats," Journal of Visualized Experiments, vol. 155, 2020.

[9] D. N. Xanthos and T. J. Coderre, "Sympathetic vasoconstrictor antagonism and vasodilatation relieve mechanical allodynia in rats with chronic postischemia pain," The Journal of Pain, vol. 9, pp. 423-433, 2008.

[10] Q. Hu, X. Zheng, X. Li et al., "Electroacupuncture alleviates mechanical allodynia in a rat model of complex regional pain syndrome type-I via suppressing spinal CXCL12/CXCR4 signaling," The Journal of Pain, vol. 21, pp. 1060-1074, 2020.

[11] Q. Hu, Q. Wang, C. Wang et al., "TRPV1 channel contributes to the behavioral hypersensitivity in a rat model of complex regional pain syndrome type 1," Frontiers in Pharmacology, vol. 10, p. 453, 2019.

[12] R. Chen, C. Yin, Q. Hu et al., "Expression profiling of spinal cord dorsal horn in a rat model of complex regional pain syndrome type-I uncovers potential mechanisms mediating pain and neuroinflammation responses," Journal of Neuroinflammation, vol. 17, 2020.

[13] J. Z. Klafke, M. A. da Silva, M. F. Rossato et al., "Acute and chronic nociceptive phases observed in a rat hind paw ischemia/reperfusion model depend on different mechanisms," Pflügers Archiv, vol. 468, no. 2, pp. 229-241, 2016.

[14] Y. Tang, L. Liu, D. Xu et al., "Interaction between astrocytic colony stimulating factor and its receptor on microglia mediates central sensitization and behavioral hypersensitivity in chronic post ischemic pain model," Brain, Behavior, and Immunity, vol. 68, pp. 248-260, 2018.

[15] H. T. Hsiao, Y. C. Lin, J. C. Wang, Y. C. Tsai, and Y. C. Liu, "Hypoxia inducible factor- $1 \alpha$ inhibition produced antiallodynia effect and suppressed inflammatory cytokine production in early stage of mouse complex regional pain syndrome model," Clinical and Experimental Pharmacology of Physiology, vol. 43, no. 3, pp. 355-359, 2016.

[16] S. Bruehl and D. S. Warner, "An update on the pathophysiology of complex regional pain syndrome," Anesthesiology, vol. 113, no. 3, pp. 713-725, 2010.

[17] W. S. Kingery, "A critical review of controlled clinical trials for peripheral neuropathic pain and complex regional pain syndromes," Pain, vol. 73, pp. 123-139, 1997.

[18] X. Wei, L. He, J. Liu et al., "Electroacupuncture for reflex sympathetic dystrophy after stroke: a meta-analysis," Journal of Stroke and Cerebrovascular Diseases, vol. 28, pp. 1388-1399, 2019.

[19] J. Wang, X. Zheng, B. Liu et al., "Electroacupuncture alleviates mechanical allodynia of a rat model of CRPS-I and modulates gene expression profiles in dorsal root ganglia," Frontiers in Neurology, vol. 11, 2020.

[20] W. Chai, Y. Tai, X. Shao et al., "Electroacupuncture alleviates pain responses and inflammation in a rat model of acute gout arthritis," Evidence-based Complementary and Alternative Medicine, vol. 2018, Article ID 2598975, 15 pages, 2018.

[21] W. J. Dixon, "Efficient analysis of experimental observations," Annual Review of Pharmacology and Toxicology, vol. 20, pp. 441-462, 1980.

[22] S. R. Chaplan, F. W. Bach, J. W. Pogrel, J. M. Chung, and T. L. Yaksh, "Quantitative assessment of tactile allodynia in the rat paw," Journal of Neuroscience Methods, vol. 53, pp. 55-63, 1994. 
[23] C. Yin, B. Liu, P. Wang et al., "Eucalyptol alleviates inflammation and pain responses in a mouse model of gout arthritis," British Journal of Pharmacology, vol. 177, pp. 2042-2057, 2020.

[24] Y. Samuni, S. Goldstein, O. M. Dean, and M. Berk, "The chemistry and biological activities of $\mathrm{N}$-acetylcysteine," Biochimica et Biophysica Acta, vol. 1830, pp. 4117-4129, 2013.

[25] W. Chen, H. Ding, X. Zhou, H. Lin, and K. C. Chou, "iRNA(m6A)-PseDNC: identifying N6-methyladenosine sites using pseudo dinucleotide composition," Analytical Biochemistry, vol. 561-562, 2018.

[26] B. Liu, Y. Tai, B. Liu, A. I. Caceres, C. Yin, and S. E. Jordt, "Transcriptome profiling reveals Th2 bias and identifies endogenous itch mediators in poison ivy contact dermatitis," JCI Insight, vol. 4, no. 14, 2019.

[27] C. Yin, B. Liu, Y. Li et al., "IL-33/ST2 induces neutrophildependent reactive oxygen species production and mediates gout pain," Theranostics., vol. 10, pp. 12189-12203, 2020.

[28] G. de Guglielmo, M. Kallupi, M. B. Pomrenze et al., "Inactivation of a CRF-dependent amygdalofugal pathway reverses addiction-like behaviors in alcohol-dependent rats," Nature Communications, vol. 10, p. 1238, 2019.

[29] D. A. Gimbel, H. B. Nygaard, E. E. Coffey et al., "Memory impairment in transgenic Alzheimer mice requires cellular prion protein," The Journal of Neuroscience, vol. 30, pp. 6367-6374, 2010.

[30] S. Yu, D. M. Doycheva, M. Gamdzyk et al., "Activation of MC1R with BMS-470539 attenuates neuroinflammation via cAMP/ PKA/Nurr1 pathway after neonatal hypoxic-ischemic brain injury in rats," Journal of Neuroinflammation, vol. 18, 2021.

[31] S. Yu, S. R. Kim, K. Jiang et al., "Quercetin reverses cardiac systolic dysfunction in mice fed with a high-fat diet: role of angiogenesis," Oxidative Medicine and Cellular Longevity, vol. 2021, Article ID 8875729, 11 pages, 2021.

[32] B. Liu, Y. Tai, A. I. Caceres et al., "Oxidized phospholipid OxPAPC activates TRPA1 and contributes to chronic inflammatory pain in mice," PLoS One, vol. 11, 2016.

[33] Y. Li, C. Yin, B. Liu et al., "Transcriptome profiling of long noncoding RNAs and mRNAs in spinal cord of a rat model of paclitaxel-induced peripheral neuropathy identifies potential mechanisms mediating neuroinflammation and pain," Journal of Neuroinflammation, vol. 18, p. 48, 2021.

[34] B. Liu, J. Escalera, S. Balakrishna et al., "TRPA1 controls inflammation and pruritogen responses in allergic contact dermatitis," The FASEB Journal, vol. 27, pp. 3549-3563, 2013.

[35] B. Liu, J. E. Linley, X. Du et al., "The acute nociceptive signals induced by bradykinin in rat sensory neurons are mediated by inhibition of M-type $\mathrm{K}^{+}$channels and activation of $\mathrm{Ca}^{2+}$-activated Cl- channels," The Journal of Clinical Investigation, vol. 120, pp. 1240-1252, 2010.

[36] Y. Li, C. Yin, X. Li et al., "Electroacupuncture alleviates paclitaxel-induced peripheral neuropathic pain in rats via suppressing TLR4 signaling and TRPV1 upregulation in sensory neurons," International Journal of Molecular Sciences, vol. 20, 2019.

[37] X. Zheng, Y. Tai, D. He et al., "ETAR and protein kinase A pathway mediate ET-1 sensitization of TRPA1 channel: a molecular mechanism of ET-1-induced mechanical hyperalgesia," Molecular Pain, vol. 15, 2019.

[38] A. S. Kaushik, L. J. Strath, and R. E. Sorge, "Dietary interventions for treatment of chronic pain: oxidative stress and inflammation," Pain and Therapy, vol. 9, pp. 487-498, 2020.
[39] A. Gornicka, G. Morris-Stiff, S. Thapaliya, B. G. Papouchado, M. Berk, and A. E. Feldstein, "Transcriptional profile of genes involved in oxidative stress and antioxidant defense in a dietary murine model of steatohepatitis," Antioxidants \& Redox Signaling, vol. 15, pp. 437-445, 2011.

[40] R. R. Ji, Z. Z. Xu, and Y. J. Gao, "Emerging targets in neuroinflammation-driven chronic pain," Nature Reviews. Drug Discovery, vol. 13, pp. 533-548, 2014.

[41] X. Li, M. Li, L. Tian, J. Chen, R. Liu, and B. Ning, "Reactive astrogliosis: implications in spinal cord injury progression and therapy," Oxidative Medicine and Cellular Longevity, vol. 2020, Article ID 9494352, 14 pages, 2020.

[42] A. J. Vickers, E. A. Vertosick, G. Lewith et al., "Acupuncture for chronic pain: update of an individual patient data metaanalysis," The Journal of Pain, vol. 19, pp. 455-474, 2018.

[43] R. R. Ji, A. Chamessian, and Y. Q. Zhang, "Pain regulation by non-neuronal cells and inflammation," Science, vol. 354, pp. 572-577, 2016.

[44] W. Wang and A. K. Jaiswal, "Nuclear factor Nrf2 and antioxidant response element regulate NRH:quinone oxidoreductase 2 (NQO2) gene expression and antioxidant induction," Free Radical Biology \& Medicine, vol. 40, pp. 1119-1130, 2006.

[45] T. Hussain, B. Tan, Y. Yin, F. Blachier, M. C. Tossou, and $\mathrm{N}$. Rahu, "Oxidative stress and inflammation: what polyphenols can do for us?," Oxidative Medicine and Cellular Longevity, vol. 2016, Article ID 7432797, 9 pages, 2016.

[46] D. Zhao, D. F. Han, S. S. Wang, B. Lv, X. Wang, and C. Ma, "Roles of tumor necrosis factor- $\alpha$ and interleukin-6 in regulating bone cancer pain via TRPA1 signal pathway and beneficial effects of inhibition of neuro-inflammation and TRPA1," Molecular Pain, vol. 15, 2019.

[47] E. Nummenmaa, M. Hämäläinen, L. J. Moilanen et al., “Transient receptor potential ankyrin 1 (TRPA1) is functionally expressed in primary human osteoarthritic chondrocytes," Arthritis Research \& Therapy, vol. 18, p. 185, 2016.

[48] A. L. Oaklander and S. H. Horowitz, "The complex regional pain syndrome," Handbook of Clinical Neurology, vol. 131, pp. 481-503, 2015.

[49] T. J. Coderre and G. J. Bennett, "A hypothesis for the cause of complex regional pain syndrome-type I (reflex sympathetic dystrophy): pain due to deep-tissue microvascular pathology," Pain Medicine, vol. 11, pp. 1224-1238, 2010.

[50] O. A. Fulas, A. Laferriere, R. S. Stein, D. S. Bohle, and T. J. Coderre, "Topical combination of meldonium and $\mathrm{N}$-acetyl cysteine relieves allodynia in rat models of CRPS-1 and peripheral neuropathic pain by enhancing NO-mediated tissue oxygenation," Journal of Neurochemistry, vol. 152, pp. 570584, 2020.

[51] J. M. Park, C. K. Kim, H. C. Lee et al., “Antiallodynic effects of vitamin $\mathrm{C}$ and vitamin $\mathrm{E}$ in chronic post-ischemia pain rat model," Korean Journal of Anesthesiology, vol. 65, pp. 442-448, 2013.

[52] S. G. Fischer, R. S. Perez, J. Nouta, W. W. Zuurmond, and P. G. Scheffer, "Oxidative stress in complex regional pain syndrome (CRPS): no systemically elevated levels of malondialdehyde, F2-isoprostanes and $8 \mathrm{OHdG}$ in a selected sample of patients," International Journal of Molecular Sciences, vol. 14, pp. 77847794, 2013.

[53] G. Chen, X. Luo, M. Y. Qadri, T. Berta, and R. R. Ji, "Sexdependent glial signaling in pathological pain: distinct roles 
of spinal microglia and astrocytes," Neuroscience Bulletin, vol. 34, pp. 98-108, 2018.

[54] R. E. Sorge, J. C. Mapplebeck, S. Rosen et al., "Different immune cells mediate mechanical pain hypersensitivity in male and female mice," Nature Neuroscience, vol. 18, no. 8, pp. 1081-1083, 2015.

[55] H. Kim, C. H. Lee, S. H. Kim, and Y. D. Kim, "Epidemiology of complex regional pain syndrome in Korea: an electronic population health data study," PLoS One, vol. 13, 2018.

[56] D. A. Andersson, C. Gentry, S. Moss, and S. Bevan, "Transient receptor potential A1 is a sensory receptor for multiple products of oxidative stress," The Journal of Neuroscience, vol. 28, pp. 2485-2494, 2008.

[57] M. Trevisani, J. Siemens, S. Materazzi et al., "4-Hydroxynonenal, an endogenous aldehyde, causes pain and neurogenic inflammation through activation of the irritant receptor TRPA1," Proceedings of the National Academy of Sciences of the United States of America, vol. 104, pp. 13519-13524, 2007.

[58] A. Bittar, J. Jun, J. H. La, J. Wang, J. W. Leem, and J. M. Chung, "Reactive oxygen species affect spinal cell type-specific synaptic plasticity in a model of neuropathic pain," Pain, vol. 158, pp. 2137-2146, 2017.

[59] Y. Shi, S. Yuan, and S. J. Tang, "Reactive oxygen species (ROS) are critical for morphine exacerbation of HIV-1 gp120induced pain," Journal of Neuroimmune Pharmacology, vol. 16, pp. 581-591, 2021.

[60] J. Zheng, J. Zhang, X. Zhang et al., "Reactive oxygen species mediate low back pain by upregulating substance $P$ in intervertebral disc degeneration," Oxidative Medicine and Cellular Longevity, vol. 2021, Article ID 6681815, 11 pages, 2021.

[61] L. Teixeira-Santos, A. Albino-Teixeira, and D. Pinho, "Neuroinflammation, oxidative stress and their interplay in neuropathic pain: focus on specialized pro-resolving mediators and NADPH oxidase inhibitors as potential therapeutic strategies," Pharmacological Research, vol. 162, 2020.

[62] Y. Q. Zhou, D. Q. Liu, S. P. Chen et al., "Reactive oxygen species scavengers ameliorate mechanical allodynia in a rat model of cancer-induced bone pain," Redox Biology, vol. 14, pp. 391$397,2018$.

[63] Y. Xue, S. Dai, J. Liang, and W. Ji, "Effect of reactive oxygen species of the psoas major muscle in complete Freund's adjuvant-induced inflammatory pain in rats," Molecular Pain, vol. 16, 2020.

[64] L. Pang, X. Lian, H. Liu et al., "Understanding diabetic neuropathy: focus on oxidative stress," Oxidative Medicine and Cellular Longevity, vol. 2020, Article ID 9524635, 13 pages, 2020.

[65] O. Pol, "The role of carbon monoxide, heme oxygenase 1, and the Nrf2 transcription factor in the modulation of chronic pain and their interactions with opioids and cannabinoids," Medicinal Research Reviews, vol. 41, pp. 136-155, 2021.

[66] J. Sun, J. Y. Li, L. Q. Zhang et al., "Nrf2 Activation Attenuates Chronic Constriction Injury-Induced Neuropathic Pain via Induction of PGC-1 $\alpha$-Mediated Mitochondrial Biogenesis in the Spinal Cord," Oxidative Medicine and Cellular Longevity, vol. 2021, Article ID 9577874, 17 pages, 2021.

[67] Y. Q. Zhou, W. Mei, X. B. Tian, Y. K. Tian, D. Q. Liu, and D. W. Ye, "The therapeutic potential of Nrf2 inducers in chronic pain: evidence from preclinical studies," Pharmacology \& Therapeutics, vol. 225, 2021.

[68] X. Zhao, L. Liu, Y. Wang, G. Wang, Y. Zhao, and Y. Zhang, "Electroacupuncture enhances antioxidative signal pathway and attenuates neuropathic pain induced by chemotherapeutic paclitaxel," Physiological Research, vol. 68, pp. 501-510, 2019.

[69] R. Jancalek, "Signaling mechanisms in mirror image pain pathogenesis," Annals of Neurosciences, vol. 18, pp. 123-127, 2011.

[70] H. Nie, B. Liu, C. Yin et al., "Gene expression profiling of contralateral dorsal root ganglia associated with mirror-image pain in a rat model of complex regional pain syndrome typeI," Journal of Pain Research, vol. 14, pp. 2739-2756, 2021.

[71] C. F. Cheng, J. K. Cheng, C. Y. Chen et al., "Mirror-image pain is mediated by nerve growth factor produced from tumor necrosis factor alpha-activated satellite glia after peripheral nerve injury," Pain, vol. 155, pp. 906-920, 2014.

[72] G. Groeneweg, F. J. Huygen, T. J. Coderre, and F. J. Zijlstra, "Regulation of peripheral blood flow in complex regional pain syndrome: clinical implication for symptomatic relief and pain management," BMC Musculoskeletal Disorders, vol. 10, no. 1, 2009.

[73] T. L. Clanton, "Hypoxia-induced reactive oxygen species formation in skeletal muscle," Journal of Applied Physiology, vol. 102, no. 6, pp. 2379-2388, 2007.

[74] G. Groeneweg, F. J. Huygen, S. P. Niehof et al., "Effect of tadalafil on blood flow, pain, and function in chronic cold complex regional pain syndrome: a randomized controlled trial," BMC Musculoskeletal Disorders, vol. 9, no. 1, 2008.

[75] M. Koban, S. Leis, S. Schultze-Mosgau, and F. Birklein, "Tissue hypoxia in complex regional pain syndrome," Pain, vol. 104, pp. 149-157, 2003. 